\title{
Recent results on functional equations in a single variable, perspectives and open problems
}

\author{
Karol BARon AND Witold JARCZYK \\ To the memory of Professor Marek Kuczma
}

Mathematics Subject Classification (2000). Primary 39-02, 39B12, 39B62; Secondary 26A18, 26A27, 26A30, 26A46, 28A05, 28C20, 30D05, 33B15, 42A32, 43A05, 60B15, 85A99, 86A10, 92B20.

Keywords. Iterative functional equations, iterative roots, convolution equations, simultaneous functional equations, Schröder's equation, Siegel's set, Abel's equation.

Summary. In 1990 Cambridge University Press published the by now well-known monograph Iterative Functional Equations by Marek Kuczma, Bogdan Choczewski and Roman Ger. In the paper we will pay attention mainly on, we think, important papers published in the last decade or not discussed in this book. Of course, the selection we made reflects our personal preferences only.

The topics of this paper are: 1 . A view of the theory developed up to now; 2. Iterative roots; 3. Functional equations with superpositions of the unknown function; 4. Some linear and convolution equations; 5. Schilling's problem; 6. Daróczy's equation; 7. Simultaneous functional equations; 8. Schröder's equation; 9. Abel's equation; 10. Miscellaneous results and problems.

In 1990 Cambridge University Press published the by now well-known monograph Iterative Functional Equations by Marek Kuczma, Bogdan Choczewski and Roman Ger [153]. In the paper we would like to pay attention mainly on, we think, important papers published in the last decade or not discussed in this book. Of course, the selection we made reflects our personal preferences only.

\section{Contents}

1. A view of the theory developed up to now

2. Iterative roots

3. Functional equations with superpositions of the unknown function

4. Some linear and convolution equations

5. Schilling's problem 
6. Daróczy's equation

7. Simultaneous functional equations

8. Schröder's equation

9. Abel's equation

10. Miscellaneous results and problems

\section{A view of the theory developed up to now}

It is our feeling that since eighties a number of results has been appearing which put some lights on certain assumptions and methods of the theory of iterative functional equations developed by Marek Kuczma and his school. In particular, we have in mind the role of the fixed points theorems of Banach and Schauder. This is the main object of a series of papers by J. Matkowski and co-authors, devoted to Lipschitzian Nemitskii operators associated with the equation

$$
\varphi(x)=h(x, \varphi[f(x)]) ;
$$

see [153; Section 5.5B]. (Concerning superposition operators see the monograph [5] by J. Appell and P. P. Zabrejko.)

Furthermore we are interested in the role played by a family of functions $f$, classical in the theory of (1.1) and of

$$
\varphi(f(x))=g(x, \varphi(x))
$$

described by the following assumption:

(H) The function $f$ maps continuously a real interval $X$ into itself and $0<$ $(f(x)-\xi) /(x-\xi)<1$ holds for every $x \in X \backslash\{\xi\}$ with a $\xi \in X$.

This assumption implies that $\xi$ is the unique fixed point of $f$ and the sequence $\left(f^{n}: n \in \mathbb{N}\right)$ of the iterates tends to $\xi$ uniformly on compact subsets of $X$. On one side this assumption made it possible to develop a theory which turned out to be also naturally applicable, e.g. to branching processes (see [153; Chapter 2]). On the other hand, in a sense, such an assumption has to occur in this theory which can be seen from [116, Corollary 2] (see also [153; Remark 5.2.6]). Under the assumption $(\mathrm{H})$ it is natural to study local solutions in a neighbourhood of the fixed point $\xi$. Otherwise one has to consider solutions in a neighbourhood of a point $x_{0}$ and its value $f\left(x_{0}\right)$; see e.g. [34] and [35] by G. R. Belitskii and V. A. Tkachenko.

Hypothesis $(\mathrm{H})$ expresses very simple dynamics of $f$. It has only one cycle which simply forms the globally attractive fixed point. Conditions characterizing such a behaviour may be found in [120]. This simple dynamics plays really a crucial role in the theory. For the linear equation

$$
\varphi(f(x))=G(x) \varphi(x)+H(x)
$$


it follows from the following theorem of B. Gawel [92; Theorem 2].

Theorem 1.1. Assume $X$ is a real interval and $Y$ is a non-degenerate Banach space. Let $f: X \rightarrow X$ and $G: X \rightarrow \mathbb{R}$ be continuous functions such that

$$
\begin{aligned}
& |G(x) G(f(x))|<1 \quad \text { for } \quad x \in \operatorname{Per}\left(f^{2}, 1\right), \\
& G(x) \neq 0 \quad \text { for } \quad x \in \bigcup_{n=1}^{\infty}\left(f^{n}\right)^{-1}(\operatorname{Per}(f, 2)) .
\end{aligned}
$$

Suppose also that the set of all continuous $H: X \rightarrow Y$ such that (1.3) has a continuous solution $\varphi: X \rightarrow Y$ is of the second category in the space $C(X, Y)$. Then $f$ can have only cycles of orders 2 and 1.

In particular, every point $x \in X$ is asymptotically periodic: there exists a $p \in$ Per $f$ such that $\lim _{n \rightarrow \infty}\left(f^{n}(x)-f^{n}(p)\right)=0$.

The dynamics of $f$ is strictly connected not only with the existence but also with the uniqueness of solutions. For instance, under some assumptions on $g$, if $f: X \rightarrow X$ is continuous, Per $f \neq \emptyset$ and $\operatorname{cl}_{X}(X \backslash[\inf \operatorname{Per} f$, sup Per $f])$ contains no cycle of $f$ of order not greater than 2 then (1.2) has at most one continuous solution $\varphi: X \rightarrow Y$ [90; Corollary 4].

Consider now the inequalities

$$
\psi(f(x)) \leq \beta(x, \psi(x))
$$

and

$$
\alpha(x, \psi(x)) \leq \psi(f(x)) \leq \beta(x, \psi(x))
$$

where $\alpha$ and $\beta$ have the following properties:

$\left(\mathrm{H}_{1}\right) \quad$ The function $\beta$ maps $X \times[0, \infty)$ into $[0, \infty), \beta(x, 0)=0$ for $x \in X$ and $\beta(x, y)<y$ for $x \in X, y \in(0, \infty)$.

$\left(\mathrm{H}_{2}\right) \quad$ The function $\alpha$ maps $X \times[0, \infty)$ into $[0, \infty), \alpha(x, 0)=0$ for $x \in X$ and $\alpha(x, y)>0$ for $x \in X, y \in(0, \infty)$.

Surprisingly the uniqueness of non-negative continuous solutions of (1.4) and (1.5) depends strongly on the compactness of $X$. To present two results of B. Gaweł $[91$; Theorems 1, 2] in this direction let us introduce two function classes. By $\mathcal{F}_{1}(X)$ denote the set of all functions $f \in C(X, X)$ such that for every $\beta$ fulfilling $\left(\mathrm{H}_{1}\right)$ the only non-negative continuous solution $\psi: X \rightarrow \mathbb{R}$ of (1.4) is the zero function. Similarly, the symbol $\mathcal{F}_{2}(X)$ will stand for the set of all $f \in C(X, X)$ with the property that for all $\alpha$ and $\beta$ fulfilling $\left(\mathrm{H}_{1}\right)$ and $\left(\mathrm{H}_{2}\right)$, the zero function is the only non-negative continuous solution $\psi: X \rightarrow \mathbb{R}$ of (1.5). Notice that $\mathcal{F}_{1}(X) \subset \mathcal{F}_{2}(X)$.

Theorem 1.2. If the interval $X$ is compact then the sets $\mathcal{F}_{1}(X)$ and $C(X, X) \backslash$ $\mathcal{F}_{2}(X)$ are nowhere dense in $C(X, X)$. 
Theorem 1.3. If the interval $X$ is not compact then the set $\mathcal{F}_{1}(X)$ is a residual subset of $C(X, X)$ and the set $C(X, X) \backslash \mathcal{F}_{2}(X)$ is a dense subset of this space.

An answer to a similar problem for equations is not known. It would be interesting to develop also such a qualitative study for other classes of functions, e.g. differentiable ones. In this direction see the paper [71] by K. Dankiewicz. Another class of functions has been studied in this spirit in [20]. Also measuretheoretical analogues of the above mentioned and other theorems are required. Measure-theoretical means here with respect to the $\sigma$-ideals of Christensen [64], or Aronszajn [6], or Gaussian which according to [70] by M. Csörnyei coincides with the Aronszajn one; cf. also [115]. In this direction only a few results may be found in the literature (see [13], [92], [198] and the next section).

\section{Iterative roots}

Solving functional equations with superpositions of the unknown function is, no doubts, rather difficult. In particular, this concerns finding iterative roots. A theoretical explanation of this experience is contained in the paper [114] by P. D. Humke and M. Laczkovich. The following is proved there.

Theorem 2.1. For every integer $N \geq 2$ the set of all continuous functions $f$ : $[0,1] \rightarrow[0,1]$ such that the equation

$$
\varphi^{N}=f
$$

has a continuous solution $\varphi:[0,1] \rightarrow[0,1]$ is an analytic and non-Borel subset of $C([0,1], \mathbb{R})$. The same property holds for the set of all continuous and increasing functions $f:[0,1] \rightarrow[0,1]$ such that $(2.1)$ has a continuous solution, and, if $N$ is odd, for the set of all continuous and decreasing functions $f:[0,1] \rightarrow[0,1]$ such that (2.1) has a continuous solution. If $N$ is even then the set of all continuous and decreasing functions $f:[0,1] \rightarrow[0,1]$ such that $(2.1)$ has a continuous solution is an $\mathcal{F}_{\sigma}$ subset of $C([0,1], \mathbb{R})$.

Another explanation is given by the following theorem of K. Simon [237; Theorem 1], [238].

Theorem 2.2. For every integer $N \geq 2$ the set of all continuous functions $f:[0,1] \rightarrow[0,1]$ such that $(2.1)$ has a continuous solution $\varphi:[0,1] \rightarrow[0,1]$ is both of first category in $C([0,1],[0,1])$ and of Wiener measure zero.

The topological part of this theorem has been strengthened by A. M. Blokh [47; Theorem 2]: 
Theorem 2.3. The set of all continuous functions $f:[0,1] \rightarrow[0,1]$ such that for some integer $N \geq 2$ equation $(2.1)$ has a continuous solution $\varphi:[0,1] \rightarrow[0,1]$ is nowhere dense in $C([0,1],[0,1])$.

According to what we have indicated above one should not expect results on iterative roots in a general situation. In fact, even iterative roots of polynomials are not described. Even worse: we do not know whether every complex cubic polynomial has a square root. In the case of quadratic polynomials we know, due to R. E. Rice, B. Schweizer and A. Sklar [217], that they have no square roots. As follows from [62; Theorem 2] by B. Choczewski and M. Kuczma they have no roots at all. Regarding the real case for quadratic polynomials see [56] by E. N. Bronshteǐn. There is a characterization, given by B. Schweizer and A. Sklar in [227], of real (complex) polynomials of order 4 having a polynomial square root. The result reads as follows.

Theorem 2.4. A polynomial of the form

$$
a_{4} x^{4}+a_{3} x^{3}+a_{2} x^{2}+\left(a_{1}+1\right) x+a_{0}, \quad a_{4} \neq 0,
$$

has a quadratic polynomial as a square iterative root if and only if there is a cube root $\gamma$ of $a_{4}$ such that

$$
4 a_{4} A_{4}=A_{2}^{2}+2 \gamma A_{2} \quad \text { and } \quad A_{3}=-1,
$$

where

$$
A_{m}=\sum_{k=0}^{m} \frac{(-1)^{k}}{4^{k}}\left(\begin{array}{c}
4-m+k \\
k
\end{array}\right)\left(\frac{a_{3}}{a_{4}}\right)^{k} a_{4-m+k}
$$

for $m \in\{0,1,2,3,4\}$.

The situation is completely clear in the case of Čebyšev polynomials. The following is proved in [216] by R. E. Rice.

Theorem 2.5. The $n$-th Čebyšev polynomial has a square root if and only if $n \equiv 0$ or $n \equiv 1(\bmod 4)$.

Regarding some other problems connected with iterative roots of polynomials see e.g. [60], [86], [182], and [216]. Roots of $1 / x$, both real and complex, are studied in [59]; the question being the title of this paper was posed by T. Ważewski and answered by St. Łojasiewicz who in [170] described the general solution of (2.1) for an arbitrary bijection $f$. Iterative roots of piecewise monotone functions have been recently studied by A. Blokh, E. Coven, M. Misiurewicz and Z. Nitecki [46]. The same paper shows also that even in the class of piecewise linear functions equation (2.1) leads to non-trivial questions. Note also the paper [166] by Z. Leśniak which 
contains a construction of continuous roots of Sperner homeomorphisms of the plane.

The problem of the existence and uniqueness of continuous iterative roots of homeomorphisms of the circle with no periodic points is considered by M. C. Zdun in [271]. It turns out that if such a homeomorphism has continuous iterative roots of $n$-th order then either there are exactly $n$ of them, or they depend on an arbitrary function.

For other problems concerning iterative roots as well as some new ideas in this field, for instance so-called phantom roots, the reader is referred to the survey paper [249] by Gy. Targonski and the references therein; cf. also [203], [204] by T. Powierża and [28] by L. Bartłomiejczyk. Many results and references concerning both formal and holomorphic roots, embeddability and linearization of (germs of) holomorphic mappings can be found in the paper [48] by S. Bogatyi.

\section{Functional equations with superpositions of the unknown func- tion}

Equation (2.1) is a special case of

$$
\sum_{j=0}^{N} a_{j} \varphi^{j}(x)=F(x) .
$$

Some other particular equations of this form have been studied for years; see [81; p. B.16]. Classical problems as existence, uniqueness, continuous dependence on given functions, and stability have been considered in several function classes by a group of Chinese mathematicians: Li Ren Zhao [279], Weinian Zhang [276][278], Jian Guo Si [234], and Jian Hua Zheng [280]; see also the survey paper [275] by Jingzhong Zhang, Lu Yang and Weinian Zhang. By using some summability methods solutions to a special case of (3.1) have been given by M. Malenica [171], [172]. In [39] by D. Bessis, S. Marmi and G. Turchetti the reader may find some applications of an equation of type (3.1) to the normal forms arising in the study of parabolic Julia sets. A. Boutabaa in [54] and [55] considers such type of equations with several unknown functions.

We would like to call reader's attention to the homogeneous equation

$$
\sum_{j=0}^{N} a_{j} \varphi^{j}(x)=0 .
$$

In the classes of bijections or continuous mappings of a real interval it was considered by A. Mukherjea and J. S. Ratti [195], J. S. Ratti and Y. F. Lin [212], and P. J. McCarthy [179]. The general solution on subsets of the positive or negative half-line was found in [126] in the case where $a_{0}=-1$ and $a_{j} \geq 0$ for 
$j \in\{1, \ldots, N\}$. More general situation is covered by the following result of J. Tabor and J. Tabor [247; Theorem 1]; see also [124].

Denote by $\Lambda$ the set of all complex roots of

$$
\sum_{j=0}^{N} a_{j} \lambda^{j}=0 .
$$

Theorem 3.1. Let $a_{0}, \ldots, a_{N}$ be real numbers and $c \in \Lambda \cap(0, \infty)$. Assume that:

(i) $c$ is a simple root of (3.3),

(ii) $(\Lambda \backslash\{c\}) \cap(0, \infty)=\emptyset$,

(iii) if $\lambda \in \Lambda \backslash\{c\}$ then $|\lambda|>c$.

If either $D \subset(-\infty, 0)$, or $D \subset(0, \infty)$, and $\varphi: D \rightarrow D$ is a solution of (3.2) then $c D \subset D$ and $\varphi(x)=c x$ for every $x \in D$.

As follows from [246] by Jacek Tabor the above $D$ may be, in fact, an arbitrary subset of a closed convex cone $K$ contained in a Banach space and such that $K \cap(-K)=\{0\}$.

It is an open problem whether conditions (i)-(iii) are necessary for the uniqueness of the (continuous, monotonic) solution $\varphi:(0, \infty) \rightarrow(0, \infty)$ of $(3.2)$. However, according to Jacek Tabor [244] this is not the case for solutions on $(0,1)$; cf. [125] and [245].

To complete the discussion of (3.2) we mention two its generalizations in different directions. The first one is the equation of infinite order

$$
\sum_{n=1}^{\infty} a_{n} \varphi^{n}(x)=x
$$

considered recently in [127]. It is proved that if $a_{n} \geq 0$ for every $n \in \mathbb{N}$ then an iterate of each solution of (3.4) defined on a subset of the positive or negative half-line is linear. The second one is the equation

$$
P\left(x, \varphi(x), \ldots, \varphi^{N}(x)\right)=0
$$

with a non-zero polynomial $P$, considered by J.-P. Bézivin [40] who proved, among others, that every entire solution of (3.5) has to be a polynomial.

Many people were interested in the functional equation

$$
\varphi(x+\varphi(x))=\varphi(x)+\varphi(\varphi(x))
$$

expressing the additivity of $\varphi$ on its graph. For the first time this equation appeared in the book $[81 ;$ p. 3.37$]$ by J. Dhombres. It was proved by G. L. Forti [88; Theorem 1] that each continuous solution $\varphi: \mathbb{R} \rightarrow \mathbb{R}$ of (3.6) which is differentiable at zero has to be linear. Some problems and conjectures concerning this 
differentiability assumption were posed by M. Laczkovich [154] (see also [211; pp. 554-555]) and M. Sablik [219]. The problem of continuous solutions was eventually solved in [117] where the following is proved.

Theorem 3.2. If $\varphi: \mathbb{R} \rightarrow \mathbb{R}$ is a continuous solution of (3.6) then either there exist real numbers $a \geq 0$ and $b \geq 0$ such that

$$
\varphi(x)= \begin{cases}a x & \text { for } x \in(-\infty, 0) \\ b x & \text { for } x \in[0, \infty)\end{cases}
$$

or there exists a real constant $c$ such that

$$
\varphi(x)=c x \quad \text { for } x \in \mathbb{R} .
$$

A shorter proof in the case where $\varphi$ maps the half-line $[0, \infty)$ into itself is presented in [119; pp. 52-61].

Solutions $\varphi:(0, \infty) \rightarrow(0, \infty)$ of $(3.6)$ such that the function $x \mapsto \varphi(x) / x$, $x \in(0, \infty)$, is monotonic are also linear according to [173] and [174; Theorem 1] by J. Matkowski. The entire solutions were considered by C. Borelli Forti and G. L. Forti [50]. Surprisingly in this function class the problem is not completely solved.

Due to S. L. Segal [229; Theorem 2] we know that if $k \geq 2$ is an integer then the only non-constant entire solution of

$$
\varphi(z+\varphi(z))=\varphi(z) \varphi\left(z^{k}+1\right)
$$

is given by $\varphi(z)=z^{k+1}$. The more general equation

$$
\varphi(z+\varphi(z))=S(\varphi(z)) \varphi(P(z))
$$

where $S, P$ are polynomials, has only polynomials as entire solutions which follows from [229; Theorem 1].

Regarding solutions $\varphi: \mathbb{R} \rightarrow \mathbb{R}$ of (3.7) with $k=1$ :

$$
\varphi(x+\varphi(x))=\varphi(x) \varphi(x+1)
$$

let us mention the paper [228] by S. L. Segal where, among others, the following conjecture is posed: The only continuous solutions $\varphi: \mathbb{R} \rightarrow \mathbb{R}$ of (3.8) are quadratic polynomials. [177].

For some other related equations see [131] and [231], [108], [119; Theorem 2.9],

There are some problems which, although not formulated in terms of functional equations, immediately lead to them. We finish this section with a presentation 
of some of them. Invariant curves, leading to a general equation with the superposition of the unknown function, viz.

$$
\varphi(f(x, \varphi(x)))=g(x, \varphi(x))
$$

yield a classical example of such a problem which is still studied in various directions. In connection with this a number of other important functional equations also occur; see e.g. [7] by Yu. V. Azarina. However, it is our feeling that equation (3.9) is studied recently not so extensively as it should be because of its importance. We mention here only papers [27], [67], [121], [235] and note that even in special cases it still provides open problems. This is the case, for instance, with the equation

$$
\varphi(x f(\varphi(x)))=g(\varphi(x))
$$

studied for power functions $f$ and $g$ by P. Kahlig, A. Matkowska and J. Matkowski in [132]. In general case, J. Matkowski (oral communication) posed the problem to find all continuous self-mappings of $(0, \infty)$ satisfying (3.10). When $f(x)=x$ and $g(x)=c x^{2}$ some continuous solutions are known due to P. Kahlig and J. Smítal [134].

Now, let $T$ be a $t$-norm on $[0,1]$. Given $\varphi:[0,1] \rightarrow[0,1]$, the problem whether $\left\{\varphi^{n}: n \in \mathbb{N}\right\}$ is a subsemigroup of $([0,1], T)$ led C. Alsina to functional equations of the form

$$
T\left(\varphi^{n}(x), \varphi^{m}(x)\right)=\varphi^{k}(x) .
$$

In [4; Theorem 3, Corollary 3] he considered them in the class of homeomorphisms. However, in our opinion this equation is interesting by itself.

Finally we present the following theorem of L. B. González [102].

Theorem 3.3. If $g_{1}$ is a non-constant entire function and $g_{n}$ for $n \geq 2$ are entire functions which are not polynomials of degree less than or equal to one then the functions

$$
1, \quad g_{1}, \quad g_{1} \circ g_{2}, \quad g_{1} \circ g_{2} \circ g_{3}, \ldots
$$

are linearly independent.

As a simple consequence the author derives from here a result of J.P. R. Christensen and P. Fischer [65; Theorem 3] on entire solutions of the Feigenbaum equation

$$
\varphi(\varphi(\lambda z))+\lambda \varphi(z)=0 .
$$

Regarding its meromorphic solutions see [66]. The Feigenbaum equation is recently extensively studied, also from the computer science point of view, and has a very vast literature. In our opinion a special survey on it and its connections with discrete dynamics is definitely required. 


\section{Some linear and convolution equations}

In this section we are going to consider the equations of the form

$$
\sum_{j=0}^{N} A_{j}(x) \varphi\left(f_{j}(x)\right)=F(x)
$$

and some of their generalizations to infinite order.

All non-negative and Lebesgue-measurable solutions $\varphi: \mathbb{R} \rightarrow \mathbb{R}$ of

$$
\varphi(x)=\sum_{j=1}^{N} A_{j} \varphi\left(x+a_{j}\right)
$$

were determined by M. Laczkovich [155; Theorem 1] under the assumption of positivity of $A_{j}$ 's; see also [153; Proposition 6.9.4]. A more elementary proof (based on a reccurent method [18; Theorem 1] and on a result of M. Kuczma [150; Theorem 5], also [151; Theorem 17.8.2] stating that any almost Jensen convex function coincides almost everywhere with a Jensen convex function) of Laczkovich's theorem has been supplied in [119; Chapter III]; for some generalizations see [105] by M. Grinč. The paper [119] deals also with the equation

$$
\varphi(x)=\sum_{j=1}^{N} A_{j} \varphi\left(f_{j}(x)\right),
$$

more general than (4.2), under the assumption that $f_{1}, \ldots, f_{N}$ are pairwise commuting homeomorphisms of an open real interval. The same equation was studied by S. Ž. Nikčević in [197] rather from an algebraic point of view. For some problems reducing to (4.2) or related to it see [10] by J. A. Baker, [77] by G. Derfel, [129], and [274] by Wei-Bin Zeng.

Non-negative and measurable solutions of the even more general equation

$$
\varphi(x)=\int_{S} \varphi(f(x, y)) \sigma(d y),
$$

where $S \subset \mathbb{R}$ is a Borel set, $\sigma$ is a Borel measure on $S$ and $f:(a, b) \times S \rightarrow(a, b)$ is a given function $(-\infty \leq a<b \leq \infty)$, have been recently studied in [122], [123]. In the case where $S=\mathbb{R}$ or $S=[0, \infty)$ and $f(x, y)=\alpha x+y$ on $S \times S$ this is one of the problems posed by Wei-Bin Zeng [273]. The existence and uniqueness of solutions of (4.3) in a class of bounded functions have been proved by J. Morawiec [185]; see also his paper [188].

A particular case of $(4.3)$, viz. the equation

$$
\varphi(x)=\int_{S} \varphi(x+y) \sigma(d y)
$$


(called the Integrated Cauchy Functional Equation) appears in probability theory (cf. [207] and [210] where the reader can also find a vast literature devoted to (4.4)). For the first time it was considered by G. Choquet and J. Deny [63] and J. Deny [76]. We present here a very general result of Ka-Sing Lau and Wei-Bin Zeng [160; Theorem 6.2] on its non-negative and locally integrable solutions.

Let $(S,+)$ be a locally compact, separable, metrizable, abelian semigroup such that + satisfies the cancellation law. Denote by $S_{0}$ the set of all $x \in S$ which have a compact neighbourhood $U$ such that, for any $y \in S, y+U$ is a neighbourhood of $y+x$. Assume that $S_{0} \neq \emptyset$. Then $S$ is embedded into a locally compact group in which the semigroup $S_{0}$ is open. Take a Haar measure $\omega$ in this group. Let $\sigma$ be a non-zero regular Borel measure on $S$ and denote by $\mathcal{E}$ the set of all non-negative and continuous exponential functions $f: S \rightarrow \mathbb{R}$ such that

$$
\int_{S} f(y) \sigma(d y)=1 \quad \text { and }\left.\quad f\right|_{S_{0}} \neq 0 .
$$

In $\mathcal{E}$ we introduce the topology of uniform convergence on compact sets.

Theorem 4.1. If the closed subsemigroup of $S$ generated by $\operatorname{supp} \sigma$ equals $S$ and $\sigma\left(S \backslash S_{0}\right)=0$ then each non-negative and $\omega$-locally integrable solution $\varphi: S \rightarrow \mathbb{R}$ of (4.4) has the form

$$
\varphi(x)=\int_{\mathcal{E}} f(x) P(d f) \quad[\omega] \text {-a.e. on } S,
$$

where $P$ is a Borel measure on $\mathcal{E}$.

For other recent papers devoted to (4.4) and some generalizations of it see [208], [209], [232], [206], [19]. In particular, the last two of them deal with inequalities associated to (4.3) and (4.4).

To state some results of M. Pycia [206; Theorem 2 and Corollary 1] on

$$
\varphi(x) \geq \int_{S} \varphi(x+y) \sigma(d y)
$$

consider a finite Borel measure $\sigma$ on $\mathbb{R}$ with compact support which intersects both the negative and positive half-lines. Let $S$ be the closed subgroup of $\mathbb{R}$ spanned by the support of $\sigma$ and consider non-negative Borel-measurable solutions $\varphi$ of (4.5) defined on $S$. It turns out that the number of solutions strictly depends on how large is the set $\Lambda$ of all real roots of the characteristic equation

$$
\int_{S} e^{\lambda y} \sigma(d y)=1 .
$$


Note that $\Lambda$ actually has at most two elements. If it has two elements then the set of solutions of (4.5) is huge [206; Fact 2]. Otherwise, we obtain uniqueness of non-negative measurable solutions: if $\Lambda=\{\lambda\}$ then $\varphi(x)=\varphi(0) e^{\lambda x}$ a.e. on $S$; if $\Lambda=\emptyset$ then $\varphi(x)=0$ a.e. on $S$. Almost everywhere means here a.e. with respect to the Lebesgue measure in the case $S=\mathbb{R}$ and everywhere if $S$ is discrete.

The inequality

$$
\varphi(x) \leq \int_{S} \varphi(f(x, y)) \sigma(d y)
$$

is one of the subjects of [19]. Here $(S, \mathcal{S}, \sigma)$ is a probability space and $f:[0,1] \times S \rightarrow$ $[0,1]$ is a function measurable with respect to the product $\sigma$-algebra $\mathcal{B} \underline{\times} \mathcal{S}$ where $\mathcal{B}$ stands for the $\sigma$-algebra of all Borel subsets of $[0,1]$. Put $f^{1}=f$,

$$
f^{n+1}\left(x, y_{1}, \ldots, y_{n+1}\right)=f\left(f^{n}\left(x, y_{1}, \ldots, y_{n}\right), y_{n+1}\right)
$$

for $x \in[0,1]$ and $y_{1}, \ldots, y_{n+1} \in S$ and extend $f^{n}$ on $[0,1] \times S^{\mathbb{N}}$ accepting $f^{n}\left(x, y_{1}, y_{2}, \ldots\right)=f^{n}\left(x, y_{1}, \ldots, y_{n}\right)$. Assuming that the function $x \mapsto \int_{S} f(x, y) \sigma(d y)$, $x \in[0,1]$, is continuous and has no fixed point in $(0,1)$ it is proved [19; Lemma $2.1]$ that for every $x \in[0,1]$ the sequence $\left(f^{n}(x, \cdot): n \in \mathbb{N}\right)$ converges a.e. with respect to the product measure $\sigma^{\infty}$ to a measurable (with respect to the product $\sigma$-algebra) function which takes the values 0 and 1 only, and [19; Proposition 2.1] the functions $p, q:[0,1] \rightarrow[0,1]$ defined by

$$
p(x)=\sigma^{\infty}\left(\lim _{n \rightarrow \infty} f^{n}(x, \cdot)=0\right), \quad q(x)=\sigma^{\infty}\left(\lim _{n \rightarrow \infty} f^{n}(x, \cdot)=1\right)
$$

are Borel-measurable solutions of equation (4.3). It turns out [19; Theorem 2.1] that these functions control bounded solutions of (4.6):

Theorem 4.2. If $\varphi:[0,1] \rightarrow \mathbb{R}$ is a Borel-measurable and bounded solution of (4.6) then

$$
\varphi(x) \leq p(x) \max \left\{\varphi(0), \limsup _{t \rightarrow 0} \varphi(t)\right\}+q(x) \max \left\{\varphi(1), \limsup _{t \rightarrow 1} \varphi(t)\right\}
$$

for every $x \in[0,1]$.

A similar theorem is obtained there for the inequality

$$
\varphi(x) \leq \int_{S} \varphi(x+y) \sigma(d y)
$$

and these results are applied to get uniqueness of some bounded solutions of (4.3) and (4.4) as well as of their discrete versions

$$
\varphi(x)=\sum_{n=1}^{\infty} p_{n} \varphi\left(f_{n}(x)\right)
$$


and

$$
\varphi(x)=\sum_{n=1}^{\infty} p_{n} \varphi\left(x+a_{n}\right) .
$$

Now we pass to the dilation equation

$$
\varphi(x)=\sum_{j=0}^{N} c_{j} \varphi(k x-j)
$$

where $k \geq 2$ is an integer and $\sum_{j=0}^{N} c_{j}=k$. It appears, in particular, in constructing wavelets of compact supports; see the survey papers [243] by G. Strang and [111] by Ch. Heil and D. Colella. Integrable, continuous and differentiable solutions are studied especially intensively in the case $k=2$ :

$$
\varphi(x)=\sum_{j=0}^{N} c_{j} \varphi(2 x-j) .
$$

An important role is played here by the number $\Delta$ defined by

$$
\Delta=\frac{1}{2} \sum_{j=0}^{N} c_{j} .
$$

According to [74; Theorem 2.1] by I. Daubechies and J. C. Lagarias, if $|\Delta| \leq 1$ and $\Delta \neq 1$, then equation (4.7) has no non-trivial solutions in $L^{1}(\mathbb{R})$. If $\Delta=1$, then there is at most one solution $\varphi \in L^{1}(\mathbb{R})$ such that $\int_{\mathbb{R}} \varphi(x) d x=1$ and [74; Corollary 2.2] for every non-trivial solution $\varphi \in L^{1}(\mathbb{R})$ of $(4.7)$ we have $\int_{\mathbb{R}} \varphi(x) d x \neq 0$ and $\operatorname{supp} \varphi \subset[0, N]$.

The following theorem [200; Theorems 3.1 and 5.1] of Yibiao Pan concerns a special case of (4.7), viz.

$$
\varphi(x)=c_{0} \varphi(2 x)+c_{1} \varphi(2 x-1)+c_{2} \varphi(2 x-2)+c_{3} \varphi(2 x-3),
$$

and may serve as an example of results on the existence of integrable solutions of the dilation equation.

Theorem 4.3. If $c_{0}, c_{1}, c_{2}, c_{3}$ are real numbers such that

$$
c_{0}+c_{2}=c_{1}+c_{3}=1,
$$

$p \in[1, \infty)$ and

$$
\frac{1}{2}\left|c_{0}-c_{1}\right|^{p}+\frac{1}{4}\left(c_{0}^{2}+\left|c_{1} c_{3}\right|\right)^{p}+\frac{1}{4}\left(c_{3}^{2}+\left|c_{0} c_{2}\right|\right)^{p}<1,
$$

then equation (4.8) has a non-trivial compactly supported solution $\varphi$ in $L^{p}(\mathbb{R})$. 
If $c_{3}=0$ and $c_{0}+c_{1}+c_{2}=2$, then according to [159; Theorem 3.1] by Ka-Sing Lau and Jianrong Wang for $p \in[1, \infty)$ equation (4.8) has a non-trivial compactly supported solution $\varphi$ in $L^{p}(\mathbb{R})$ if and only if either $c_{1}=1$ and $\left|c_{0}\right|^{p}+\left|c_{2}\right|^{p}<2$, or $c_{0}=c_{2}=1$. In the same case, the existence of such solutions for $p \in(0, \infty)$ as well as Lebesgue-measurable solutions has been characterized by A. O. Pittenger and J. V. Ryff in [202] under the additional condition $\varphi(x)+\varphi(x+1)=1$ a.e. in $[0,1]$ (see their Theorems 4.13 and 4.7 where also formulas for the solutions are given; concerning this additional condition consult their Proposition 2.7). If $c_{3}=0, c_{2}=0$ and $c_{0} c_{1} \neq 0$, then, as follows from [202; Proposition 2.9], equation (4.8) has a non-trivial Lebesgue-measurable solution with compact support if and only if $c_{0}=c_{1}=1$.

Continuous solutions of (4.7) are constructed in [68] by D. Colella and Ch. Heil. Concerning differentiable ones see [74] by I. Daubechies and J. C. Lagarias. Various aspects of solvability of (4.7) are examined by Wang Jian Zhong in [281]. For $N=1$ irregular solutions of (4.7) have been studied by J. Morawiec in [190]. The equation

$$
\varphi(x)=\int_{\mathbb{R}} \varphi(2 x-y) \sigma(d y)
$$

is also a special case of (4.3), but more general than (4.7). It is studied in [79] by G. Derfel, N. Dyn and D. Levin in the class of normalized compactly supported distributions and (probability) distribution functions on $\mathbb{R}$. In the discrete case

$$
\varphi(x)=\sum_{n=1}^{\infty} q_{n} \varphi\left(2 x-a_{n}\right)
$$

the following is proved there (Corollary 14).

Theorem 4.4. If $\left(q_{n}: n \in \mathbb{N}\right)$ is a sequence of non-negative reals summing up to one and $\left(a_{n}: n \in \mathbb{N}\right)$ is any sequence of reals then in the class of distribution functions equation (4.9) has exactly one solution which, moreover, is a continuous function.

Assuming additionally that $q_{n}=0$ for $n \geq 7$ and $a_{n}=n-1$, A. Deliu and M. C. Spruill [75; Theorem 5.5] characterized all the $q$ 's providing absolute continuity of the solution.

A more general situation of the equation

$$
\varphi(x)=\sum_{j=1}^{N} p_{j} \int_{\mathbb{R}} \varphi\left(\alpha_{j}(x-y)\right) \sigma_{j}(d y)
$$

is studied by G. Derfel in [78]. Here $p_{1}, \ldots, p_{N}$ are non-negative reals summing up to one, $\alpha_{1}, \ldots, \alpha_{N}$ are positive reals, and $\sigma_{1}, \ldots, \sigma_{N}$ are probability Borel measures on $\mathbb{R}$. To formulate his result [78; Theorem 2] put 


$$
K=\sum_{j=1}^{N} p_{j} \log \alpha_{j}
$$

Theorem 4.5. If $K>0$ then equation (4.10) has a non-constant bounded and continuous solution $\varphi: \mathbb{R} \rightarrow \mathbb{R}$. If $K<0$ then there is no such a solution.

In fact, this theorem is a particular case of a result concerning the equation

$$
\varphi(x)=\int_{\mathbb{R} \times(0, \infty)} \varphi\left(\frac{x-y}{z}\right) \sigma(d y, d z) .
$$

In the discrete case, if $\sigma_{j}\left(\left\{-\beta_{j} / \alpha_{j}\right\}\right)=1$ for $j \in\{1, \ldots, N\}$, equation (4.10) takes the form

$$
\varphi(x)=\sum_{j=1}^{N} p_{j} \varphi\left(\alpha_{j} x+\beta_{j}\right) .
$$

Thus, if $K>0$ then equation (4.11) has a non-constant bounded and continuous solution $\varphi: \mathbb{R} \rightarrow \mathbb{R}$. However, if in addition $\beta_{1}=\ldots=\beta_{N}=0$ then the only solution $\varphi:(0, \infty) \rightarrow \mathbb{R}$ of $(4.11)$ which is bounded in a vicinity of 0 and has a limit (finite or not) at infinity is constant. This was proved by J. Ger and M. Sablik [94; Proposition 3.1]. On the other hand, as follows from [185; Corollary 1] by J. Morawiec, we have the existence and uniqueness of solutions of the equation

$$
\varphi(x)=\sum_{n=1}^{\infty} p_{n} \varphi\left(\alpha_{n} x+\beta_{n}\right)
$$

which generalizes both (4.11) and (4.9), in a class of distribution functions.

Theorem 4.6. Assume $\left(\alpha_{n}: n \in \mathbb{N}\right),\left(\beta_{n}: n \in \mathbb{N}\right)$ and $\left(p_{n}: n \in \mathbb{N}\right)$ are sequences of real numbers such that

$$
\alpha_{n}+\beta_{n} \geq 1, \quad \beta_{n} \leq 0, \quad p_{n} \geq 0 \quad \text { for every } n \in \mathbb{N}, \quad \sum_{n=1}^{\infty} p_{n}=1,
$$

and there exist positive integers $k, l$ such that

$$
\alpha_{k}+\beta_{k}=1, \quad \beta_{k}<0, \quad \alpha_{l}>1, \quad \beta_{l}=0, \quad p_{k}>0, \quad p_{l}>0 .
$$

Then equation (4.12) has exactly one solution in the class of bounded functions $\varphi: \mathbb{R} \rightarrow \mathbb{R}$ satisfying the conditions

$$
\left.\varphi\right|_{(-\infty, 0]}=0,\left.\quad \varphi\right|_{[1, \infty)}=1
$$

and this solution is continuous and increasing. 
The next theorem due to J. Morawiec [188; Corollary 2] gives some properties of the solution $\varphi$ of (4.11) obtained in Theorem 4.6. It generalizes a result of J. M. Borwein and R. Girgensohn [51; Theorem 2] and, consequently, a special case of the latter $(N=2)$ proved by S. Paganoni Marzegalli [199; Theorem 3]. Concerning the infinite case of (4.12) consult [188; Corollary 3].

Theorem 4.7. Assume $N \geq 2$ is an integer, $\alpha_{1}, \ldots, \alpha_{N}, \beta_{1}, \ldots, \beta_{N}, p_{1}, \ldots, p_{N}$ are real numbers such that

$$
\alpha_{j}+\beta_{j} \geq 1, \quad \beta_{j} \leq 0, \quad p_{j}>0 \quad \text { for every } j \in\{1, \ldots, N\}, \quad \sum_{j=1}^{N} p_{j}=1,
$$

and there exist $k, l \in\{1, \ldots, N\}$ such that

$$
\alpha_{k}+\beta_{k}=1, \quad \beta_{k}<0, \quad \alpha_{l}>1, \quad \beta_{l}=0 .
$$

(i) If $\alpha_{j}>1$ for $j \in\{1, \ldots, N\}$ and

$$
\bigcup_{j=1}^{N}\left[-\frac{\beta_{j}}{\alpha_{j}}, \frac{1-\beta_{j}}{\alpha_{j}}\right]=[0,1]
$$

then the solution of (4.11) is strictly increasing on $[0,1]$.

(ii) If (4.13) does not hold and the open intervals $\left(-\frac{\beta_{j}}{\alpha_{j}}, \frac{1-\beta_{j}}{\alpha_{j}}\right), j \in\{1, \ldots, N\}$, are pairwise disjoint then there exists a family $\mathcal{I}$ of pairwise disjoint open subintervals of $(0,1)$ such that $\varphi$ is constant on each of them and $[0,1] \backslash \cup \mathcal{I}$ is of Lebesgue measure zero.

J. M. Borwein and R. Girgensohn [51] discuss thoroughly particular cases of (4.11), too, establishing regularity properties of the solution. Also some interesting references can be found there showing that the problem has quite a long history and interesting connections with Pisot-Vijayaraghavam numbers.

Of course there is a number of other papers dealing with (4.11) and with equations of similar, in particular non-homogeneous, forms. We mention here only [224] by J. Saint-Raymond where differentiable and analytic solutions are examined, [110] by H. Haruki and Th. M. Rassias where Bernoulli polynomials are characterized, and [9] by J. A. Baker where solutions in the class of tempered distributions are sought. The existence and uniqueness of continuous solutions (defined on a compact Hausdorff space) of (4.1) with constant coefficients are studied by A. Böttcher and H. Heidler in [53].

The equation

$$
\varphi(x)=u\left(\frac{x}{2}\right) \varphi\left(\frac{x}{2}\right)+u\left(\frac{x+1}{2}\right) \varphi\left(\frac{x+1}{2}\right)
$$


with a given continuous $u:[0,1] \rightarrow[0, \infty)$ satisfying $u(x)+u\left(x+\frac{1}{2}\right)=1$ for $x \in\left[0, \frac{1}{2}\right]$ is treated by J.-P. Conze and A. Raugi in [69], in particular in a connection with wavelet theory. It turns out (Théorème 4.2) that the linear space of continuous solutions on $[0,1]$ is finite-dimensional. Moreover, under a regularity condition on $u$, the case where the space is one-dimensional is characterized.

The paper [205] by C. Praagman brings a generalization of Nörlund's result stating that given entire functions $a_{0}, \ldots, a_{N}$ there exist meromorphic solutions $\varphi_{1}, \ldots, \varphi_{N}$ on $\mathbb{C}$ of the equation

$$
\sum_{j=0}^{N} a_{j}(z) \varphi(z+j)=0,
$$

linearly independent over the periodic functions. In the real case the asymptotic behaviour of solutions of this equation is studied by M. H. Lantsman [158]. We mention here also the paper [36] by G. Belitskii and V. Tkachenko where differentiable and real analytic vector-valued solutions of (4.1) with $f_{j}$ 's being translations in a Euclidean space are examined and [191] by J. Morawiec and J. Walorski providing some its extension; see also [33] and [30]. Systems of such equations in a pretty general algebraic setting appear in [156] by M. Laczkovich where also some applications of them to studying operators commuting with the translation operators on function spaces are presented. Concerning the asymptotic behaviour of Lebesgue-measurable solutions of (4.1) defined on the real line see [11] by J. A. Baker. For some close connections between the Collatz " $3 n+1$ problem" and linear equations consult [37], [38] by L. Berg and G. Meinardus.

\section{Schilling's problem}

Studies of a physical problem have led R. Schilling [225; p. 230, eq. (52) for $\left.p=\frac{1}{2}\right]$, cf. also [80; Section 1a], to looking for solutions $\varphi: \mathbb{R} \rightarrow \mathbb{R}$ of the equation

$$
4 q \varphi(q x)=\varphi(x-1)+\varphi(x+1)+2 \varphi(x)
$$

such that

$$
\varphi(x)=0 \quad \text { for } \quad|x|>Q
$$

where $q \in(0,1)$ is fixed and

$$
Q=\frac{q}{1-q}
$$

In what follows any solution $\varphi: \mathbb{R} \rightarrow \mathbb{R}$ of (5.1) satisfying (5.2) will be called a solution of Schilling's problem.

The first non-trivial solution of (5.1) and (5.2) was found by R. Schilling for $q=\frac{1}{2}$, viz.

$$
\varphi_{1}(x)=\max \{1-|x|, 0\}
$$


Other non-trivial solutions were found for numbers of the form $q=\frac{1}{\sqrt[n]{2}}$ in $[21$; Théorème 3]:

Theorem 5.1. If $n$ is a positive integer and $q=1 / \sqrt[n]{2}$ then the convolution

$$
\varphi_{1}(x) * \varphi_{1}(q x) * \ldots * \varphi_{1}\left(q^{n-1} x\right)
$$

is a solution of Schilling's problem.

Theorems 5.2-5.6 show that $q=\frac{1}{2}$ and $q=\frac{1}{2 \sqrt{2}}$ are, in a sense, critical values for Schilling's problem. The first two come from the paper [80] by G. Derfel and R. Schilling (cf. Theorems 5a, 4 and Remark 3 there).

Theorem 5.2. For almost all $q \in\left[\frac{1}{2}, 1\right)$ there exists a non-trivial continuous solution of Schilling's problem.

Theorem 5.3. There are infinitely many $q \in\left[\frac{1}{2}, 1\right)$ such that the only continuous solution of Schilling's problem is the zero function.

If $q=\frac{\sqrt{5}-1}{2}$ then zero is the unique solution also in the following classes: one-hand-side continuous functions in a right vicinity of $-Q$ (left vicinity of $Q$ ) (cf. [186; Corollary] by J. Morawiec) and Lebesgue-integrable functions which are continuous at zero (see [21; Théorème 5]).

The next result [21; Théorème 4] implies that for $q \in\left(0, \frac{1}{2}\right)$ the problem has no non-trivial continuous (and even bounded Lebesgue-measurable) solutions.

Theorem 5.4. Assume $q \in\left(0, \frac{1}{2}\right)$. If $\varphi$ is a non-trivial Lebesgue-integrable solution of Schilling's problem then

$$
\int_{0}^{\varepsilon}|\varphi(x)|^{\frac{\log q}{\log (2 q)}} d x=\infty \quad \text { for every } \varepsilon>0
$$

On the other hand we have the following results of Y. Peres and B. Solomyak [201; Corollary 1.5].

Theorem 5.5. For almost all $q \in\left(\frac{1}{2 \sqrt{2}}, 1\right)$ there exists a non-trivial Lebesgueintegrable solution of Schilling's problem.

Theorem 5.6. If $q \in\left(0, \frac{1}{2 \sqrt{2}}\right)$ then every Lebesgue-integrable solution of Schilling's problem vanishes almost everywhere. 
It turns out that also regularity of the solution strictly depends on the parameter $q$; cf. [80; Theorem 2] by G. Derfel and R. Schilling which reads

Theorem 5.7. If Schilling's problem has a non-trivial $r$ times differentiable solution then $q \geq \frac{1}{\sqrt[r+1]{4}}$.

Another theorem due to them [80; Theorem 5b] shows that for $q$ 's close enough to 1 one can find a solution of arbitrarily high regularity:

Theorem 5.8. For every positive integer $n$ there exists a $q_{n} \in\left(\frac{1}{2}, 1\right)$ such that $\lim _{n \rightarrow \infty} q_{n}=1$ and for almost all $q \in\left(q_{n}, 1\right)$ Schilling's problem has a non-trivial $2(n-1)$ times differentiable solution.

All the results quoted above strictly depend on a range of the parameter $q$. No such restriction occurs in the next result concerning the uniqueness of solutions; cf. [22], also [74; Theorem 2.1.(b)] and [87; Corollary 3].

Theorem 5.9. The vector space of Lebesgue-integrable solutions of Schilling's problem is at most one-dimensional.

Hence, if for instance $n$ is a positive integer and $q=\frac{1}{\sqrt[n]{2}}$ then (cf. Theorem 5.1) the vector space of Lebesgue-integrable solutions is one-dimensional; if $q \in$ $\left(0, \frac{1}{2 \sqrt{2}}\right)$ then (Theorem 5.6) this space is zero-dimensional. However, for $q \in$ $\left(0, \frac{1}{3}\right]$ and for $q=\frac{1}{2}$ we have better results due to W. Förg-Rob [87; Corollary 4 and Theorems 29, 35].

Theorem 5.10. If $q \in\left(0, \frac{1}{3}\right)$ then every solution of Schilling's problem vanishes almost everywhere.

Theorem 5.11. If $q=\frac{1}{3}$ then every Lebesgue-measurable solution of Schilling's problem vanishes almost everywhere.

Theorem 5.12. If $q=\frac{1}{2}$ and $\varphi$ is a solution of Schilling's problem which is Lebesgue-measurable on a subset of $[-1,1]$ of positive Lebesgue measure then $\varphi(x)=$ $c \varphi_{1}(x)$ a.e., where $c \in \mathbb{R}$.

In the theorem above the Lebesgue-measurability of a solution on a subset of $[-1,1]$ of positive Lebesgue measure can be replaced by its continuity at at least one point of $(-1,1)$. This is known due to W. Förg-Rob [87; Section IIIc)].

The following theorem proved by R. Girgensohn and J. Morawiec in [101] shows that every non-trivial Lebesgue-integrable solution of Schilling's problem is either positive or negative. 
Theorem 5.13. If $\varphi$ is a non-trivial Lebesgue-integrable solution of Schilling's problem then either $\varphi(x)>0$ a.e. on $[-Q, Q]$ or $\varphi(x)<0$ a.e. on $[-Q, Q]$.

We have no restriction on the parameter $q$ in this theorem but according to Theorem 5.6 it concerns in fact the case where $q \geq \frac{1}{2 \sqrt{2}}$.

Regarding bounded solutions we have the following two results. For the first one see [15; Theorem 1].

Theorem 5.14. If $q \in(0, \sqrt{2}-1]$ then the zero function is the only solution of Schilling's problem which is bounded in a neighbourhood of the origin.

R. Schilling has known this for $q \in\left(0, \frac{1}{3}\right)$. J. Morawiec [184] generalized Theorem 5.14 in two directions as follows.

Theorem 5.15. If

$$
0<q<\frac{1}{3}(1-\sqrt[3]{2}+\sqrt[3]{4})
$$

then the zero function is the only solution of Schilling's problem which is bounded in a neighbourhood of at least one point of the form

$$
\varepsilon \sum_{i=1}^{m} q^{i}, \quad \text { where } \quad m \in \mathbb{N} \cup\{0, \infty\} \quad \text { and } \varepsilon \in\{-1,1\} .
$$

In [187] J. Morawiec obtained a similar result where the numbers (5.3) are replaced by numbers forming a larger set, however, the $q$ 's there are taken from a smaller set than above. In [189] he even found parameters $q \in\left(\frac{1}{3}, \frac{1}{2}\right)$ for which the zero function is the only solution bounded in a neighbourhood of a point of the interval $[-Q, Q]$.

As pointed out by J. M. Borwein and R. Girgensohn [51; Section 4] there is a close connection between Schilling's problem and the distribution function of the random series $\sum \pm q^{n}$ where the signs are chosen with probability $\frac{1}{2}$ each: The distribution function is [51; Observation] a solution of the functional equation

$$
\varphi(x)=\frac{1}{2} \varphi\left(\frac{x}{q}-\frac{1}{2}\right)+\frac{1}{2} \varphi\left(\frac{x}{q}+\frac{1}{2}\right)
$$

and if $\varphi: \mathbb{R} \rightarrow \mathbb{R}$ is an absolutely continuous solution of (5.4) with $\left.\varphi\right|_{\left(-\infty,-\frac{1}{2} Q\right]}=0$ and $\left.\varphi\right|_{\left[\frac{1}{2} Q, \infty\right)}=1$ then the convolution $\varphi^{\prime} * \varphi^{\prime}$ is a non-trivial Lebesgue-integrable solution of Schilling's problem. There does not hold a converse of this theorem: there are (cf. Theorems 5.5 and 4.7) values of $q$ such that the Schilling problem has a non-trivial Lebesgue-integrable solution but (5.4) has no absolutely continuous solution. However, no such value of $q$ is currently known explicitly. 
Besides [21] distribution solutions of (5.1) appear also in [236] by A. Simon and P. Volkmann where such a solution is found for $q=\frac{1}{4}$. For other problems see [214].

\section{Daróczy's equation}

This is the equation

$$
\varphi(x)=\varphi(x+1)+\varphi(x(x+1)) .
$$

The following problems were posed by Z. Daróczy [72].

1. Is every solution $\varphi: \mathbb{N} \rightarrow(0, \infty)$ of $(6.1)$ of the form $\varphi(n)=\frac{c}{n}$ with a constant $c$ ?

2. Determine sequences $k: \mathbb{N} \rightarrow \mathbb{N}$ such that the equation

$$
\varphi(n)=\varphi(n+1)+\varphi(k(n))
$$

has a solution $\varphi: \mathbb{N} \rightarrow(0, \infty)$.

3 . Find a weak condition under which the (positive) solution $\varphi:(0, \infty) \rightarrow \mathbb{R}$ of (6.1) is necessarily of the form

$$
\varphi(x)=\frac{c}{x}
$$

with a real constant $c$.

M. Laczkovich and R. Redheffer solved the first problem and found a large class of sequences $k$ satisfying the requirement from the second problem of Z. Daróczy proving the following [157; Corollary 1 and the commentary below it].

Theorem 6.1. Assume $k: \mathbb{N} \rightarrow \mathbb{N}$ satisfies $k(n)>n$ for every $n \in \mathbb{N}$ and

$$
k(n)-n^{2}=O\left(n^{\beta}\right)
$$

with some $\beta<2$. Then equation (6.2) has a solution $\varphi: \mathbb{N} \rightarrow(0, \infty)$ such that

$$
\liminf _{n \rightarrow \infty} n \varphi(n)=1 \quad \text { and } \quad \limsup _{n \rightarrow \infty} n \varphi(n)=3 .
$$

It seems that any condition forcing (6.3) should be connected with the behaviour of the solution $\varphi$ of (6.1) at infinity. The following result [16] (see also [14; Theorem 2]) yields a condition in this spirit.

Theorem 6.2. If $\varphi:(0, \infty) \rightarrow \mathbb{R}$ is a solution of (6.1) such that there exists a (finite or infinite) limit

$$
\lim _{x \rightarrow \infty} x \varphi(x)
$$

then $\varphi$ is of form (6.3). 
If a real function $f$ defined on a vicinity of infinity is convex then in a vicinity of infinity the function $x \mapsto f(x) / x$ is monotonic. Hence, if $\varphi:(0, \infty) \rightarrow \mathbb{R}$ is a solution of (6.1) such that $x \mapsto x^{2} \varphi(x)$ is convex in a vicinity of infinity then $\varphi$ has form (6.3). Similarly, if $\varphi:(0, \infty) \rightarrow \mathbb{R}$ is a solution of (6.1) such that $x \mapsto 1 / \varphi(x)$ is convex in a vicinity of infinity, then $x \mapsto 1 /(x \varphi(x))$ has a limit at infinity and, due to the fact that $\varphi$ has a constant sign in a vicinity of infinity, the limit (6.4) exists and $\varphi$ has form (6.3). These facts have been obtained in [118] directly. It is also proved there that the convexity of $x \mapsto \varphi(1 / x)$ in a vicinity of the origin is a sufficient condition for $\varphi$ to be of form (6.3). However, we do not know whether the convexity of the solution itself guarantees its uniqueness. If the answer was negative it would be interesting to study the problem in smaller classes, for instance for completely monotonic or logarithmically convex functions. It seems that it would be also interesting to answer the uniqueness problem for the Daróczy equation in the class of regularly varying functions in the sense of

Karamata. We emphasize here that there are very few results in the theory of functional equations involving this class of functions.

The existence of the limit $\lim _{x \rightarrow \infty} \varphi(x)$ does not imply form (6.3) of the solution. L. Bartłomiejczyk [23; Remark 3(ii)] observed that there exists a solution $\varphi$ : $(0, \infty) \rightarrow(0, \infty)$ of $(6.1)$ which is discontinuous at every point and such that $\lim _{x \rightarrow \infty} \varphi(x)=0$ and in [24; Corollary 1] he proved even more:

Theorem 6.3. For every $c_{1}, c_{2} \in[-\infty, \infty]$ with $c_{1}<c_{2}$ there exists a solution $\varphi:(0, \infty) \rightarrow \mathbb{R}$ of $(6.1)$ such that its graph is contained in the set

$$
\left\{(x, y) \in(0, \infty) \times \mathbb{R}: \quad \frac{c_{1}}{x} \leq y \leq \frac{c_{2}}{x}\right\}
$$

and meets every Borel subset of (6.5) with uncountable vertical projection.

Finally we mention also that the general solution of (6.1) may be obtained via a standard recurrent method starting from an arbitrary function defined on an interval; see [193] by Z. Moszner, also [23; Theorem 1] by L. Bartłomiejczyk.

\section{Simultaneous functional equations}

We start with two such equations found by R. Girgensohn [99], [100], to answer H.H. Kairies' question [141] whether there are functional equations which, together with regularity conditions, characterize the Minkowski singular function ?: $[0,1] \rightarrow$ $[0,1]$. According to [100; Theorem 1] the Minkowski function ? is the unique bounded solution $\varphi:[0,1] \rightarrow \mathbb{R}$ of the simultaneous functional equations

$$
\varphi\left(\frac{x}{x+1}\right)=\frac{1}{2} \varphi(x), \quad \varphi\left(\frac{1}{x+1}\right)=1-\frac{1}{2} \varphi(x) .
$$


Another system characterizing the Minkowski function can be found in G. de Rham [215; Section 4]. This is an example of a typical to some extent situation. It turns out that the unique bounded or continuous solution of simultaneous equations is often in a sense a peculiar function: for instance singular or nowhere differentiable; see e.g. the books [148; Chapter XI, $\S \S 4$ \& 5] of M. Kuczma and [153; Sections 10.5 and 10.6] by M. Kuczma, B. Choczewski and R. Ger, and the articles [73] by W. F. Darsow, M. J. Frank and H.-H. Kairies, [96]-[98] by R. Girgensohn, and the survey paper [143] by H.-H. Kairies; see also [178] by G. Mayor and J. Torrens. Some of the most prominent nowhere differentiable functions can be even characterized as solutions of such systems; see, for instance, [144] by H.-H. Kairies where Takagi's function is studied and [145] of the same author where, among others, Takagi's function and Weierstrass' function are characterized. Moreover, the non-differentiability of such functions can then be shown without making use of their actual analytical definition. C. Sempi [230] asked whether it is possible to characterize the Brownian motion via functional equations.

However, also such a regular function as Euler's $\Gamma$ may be characterized by simultaneous equations. As an example we quote here the following result by H.-H. Kairies [139; Satz 2].

Theorem 7.1. The only function $\varphi:(0, \infty) \rightarrow(0, \infty)$ satisfying

$$
\prod_{k=0}^{p-1} \varphi\left(\frac{x+k}{p}\right)=(2 \pi)^{\frac{1}{2}(p-1)} p^{\frac{1}{2}-x} \varphi(x)
$$

for all but finitely many primes $p$ and every $x \in(0, \infty)$ which is continuous on $(0,1+\varepsilon)$ for an $\varepsilon>0$ and such that $\varphi(1)=1$ is the gamma function.

The gamma function may be also characterized via a single equation (7.1). Due to H.-H. Kairies [142; Theorem 7] we know that if an integer $p \geq 2$ is fixed then $\Gamma$ is the only solution $\varphi:(0, \infty) \rightarrow(0, \infty)$ of $(7.1)$ which is absolutely continuous on $\left[\frac{1}{p}, 1+\varepsilon\right]$ for an $\varepsilon>0$ and satisfies $\lim _{x \rightarrow 0} x \varphi(x)=1$. According to [140; Satz 5] the same is true provided $\varphi$ is absolutely continuous only on $\left[\frac{1}{2}, 1+\varepsilon\right]$ but additionally $\varphi$ satisfies

$$
\varphi(x) \varphi(1-x)=\frac{\pi}{\sin \pi x} \quad \text { for every } \quad x \in(0,1) .
$$

There are also some generalizations of the classical Bohr-Mollerup characterization ([148; p. 153], [153; Section 10.4], [162], [260; Theorem 5.3.5]) of $\Gamma$ as the only normalized logarithmically convex solution $\varphi:(0, \infty) \rightarrow(0, \infty)$ of the equation

$$
\varphi(x+1)=x \varphi(x)
$$

see for instance [106] and [107] by D. Gronau and J. Matkowski, [175] by the second of them, [183] by D. S. Moak, [8] by M. Badiale, [180] by M. Merkle and 
also [261] by R. Webster, where $\Gamma$-type functions are studied more thoroughly. In [161] D. Laugwitz and B. Rodewald gave another characterization of the gamma function by use a property originally treated by Euler.

Following D. E. Knuth [146; p. 42, exercise 39 (and the answer to it on pp. 477478) and problem 40] and M. F. Yoder [266] consider now replicative functions, i.e. solutions of simultaneous equations

$$
\frac{1}{n} \sum_{k=0}^{n-1} \varphi\left(\frac{x+k}{n}\right)=a_{n} \varphi(x)+b_{n}
$$

where $a$ and $b$ are given number sequences. It turns out that in fact it is enough to examine replicativity in the case where either $b=0$ or $a=1$. In the first case in numerous classes of functions the periodic solutions are of the form

$$
c_{0}+c_{1} \sum_{n=1}^{\infty} a_{n} \mathrm{e}^{2 \pi \mathrm{i} n x}+c_{2} \sum_{n=1}^{\infty} a_{n} \mathrm{e}^{-2 \pi \mathrm{i} n x}
$$

cf. [266] by M. F. Yoder and [137] by H.-H. Kairies (see also [136]); the reader is referred to the paper [181] by J. Milnor, too. On the other hand [266; Theorem 4] aperiodic solutions may exist only if $a_{n}=n^{s}$ for some $s$. The case $a=1$ is treated by P. Schroth in [226]. Assuming $b_{n}=\log n$ he obtained uniqueness results in which the solution is expressed via the Gaussian digamma function $\Psi$.

We finish this replicativity part with a problem posed by H.-H. Kairies [138] (cf. also [135]). Characterize those complex sequences $a$ satisfying

$$
a_{m \cdot n}=a_{m} \cdot a_{n} \quad \text { for } \quad m, n \in \mathbb{N}
$$

for which there exists a non-trivial continuous function $\varphi: \mathbb{R} \rightarrow \mathbb{C}$ such that

$$
\frac{1}{n} \sum_{k=0}^{n-1} \varphi\left(\frac{x+k}{n}\right)=a_{n} \varphi(x)
$$

holds for all $x \in \mathbb{R}$ and $n \in \mathbb{N}$.

Concerning extended replicativity given by

$$
\frac{1}{n} \sum_{k=0}^{n-1} \varphi\left(\frac{x+k}{n}\right)=\sum_{j=1}^{\infty} \lambda_{n}(j) \varphi(j x)
$$

consult [167] and [168] by L.G. Lucht. Now the situation depends essentially on arithmetical properties of the sequences $\lambda_{n}$. The following question was posed by L. G. Lucht [169] in connection with the above system. Assume $\lambda: \mathbb{N} \rightarrow \mathbb{C}^{\mathbb{N}}$. 
Is it true that the $\mathbb{C}$-linear space of all its solutions $\varphi \in L^{1}(\mathbb{R} / \mathbb{Z})$ is at most two-dimensional?

Complex exponential polynomials have been characterized by J.-P. Bézivin and F. Gramain [45] via the system

$$
\sum_{m=0}^{M} P_{m}(z) \varphi(z+\alpha m)=f(z), \quad \sum_{n=0}^{N} Q_{n}(z) \varphi(z+\beta n)=g(z) .
$$

Here the $P$ 's and $Q$ 's are polynomials, $\alpha, \beta \in \mathbb{C} \backslash\{0\}$, and $f, g$ are exponential polynomials. We quote now a result of F. Gramain [104] concerning the case of constant $P$ 's and $Q$ 's.

Theorem 7.2. Let $m$ and $n$ be positive integers. If $\alpha, \beta, a_{0}, \ldots, a_{m}, b_{0}, \ldots, b_{n}$ are complex numbers such that

$$
a_{0} a_{m} b_{0} b_{n} \neq 0 \quad \text { and } \quad \frac{\alpha}{\beta} \notin \mathbb{R},
$$

then every entire solution $\varphi$ of the system

$$
\sum_{k=0}^{m} a_{k} \varphi(z+\alpha k)=0, \quad \sum_{l=0}^{n} b_{l} \varphi(z+\beta l)=0
$$

is an exponential polynomial.

For two proofs different from the original one the reader is referred to [45; Section 4].

Simultaneous equations were used by J. A. Baker [12; Theorem 2] to characterize harmonic polynomials. To formulate his result fix an integer $N \geq 2$ and denote by $\mathcal{O}_{N}$ the set of all real orthogonal $N \times N$ matrices and let $v_{0}, v_{1}, \ldots, v_{N}$ be the vertices of some fixed regular simplex in $\mathbb{R}^{N}$ with center 0 and radius 1 . Consider the system

$$
\sum_{j=0}^{N} f\left(x+r U v_{j}\right)=(N+1) f(x), \quad U \in \mathcal{O}_{N} .
$$

Theorem 7.3. If a continuous function $f: \mathbb{R}^{N} \rightarrow \mathbb{C}$ is a solution of $(7.2)$ with $r=1$, then it is a harmonic polynomial of degree at most 2 and satisfies (7.2) for all $r \in \mathbb{R}$.

Now let us call the attention of the reader to the following two papers. In the first one [252] by P. Volkmann and H. Weigel the existence of a solution $\varphi$ of the system 


$$
\varphi(f(x))=g(x, \varphi(x))
$$

(with f's and g's running over given sets of functions) is proved. The second one [176] by J. Matkowski deals with the solutions of the system

$$
\varphi(p x+q)=a \varphi(x), \quad \varphi(r x+s)=b \varphi(x)
$$

in pretty large classes of functions.

Regarding Abel simultaneous functional equations the reader is referred to Section 9.

Finally, let us recall a result of P. Volkmann [251] on simultaneous functional inequalities, one of which is of two variables

$$
\varphi(f(|x|)) \geq f(|\varphi(x)|), \quad \varphi(x+y) \geq \varphi(x)+\varphi(y) .
$$

Theorem 7.4. Assume $f:[0, \infty) \rightarrow[0, \infty)$ is continuous and strictly increasing having 0 and 1 as fixed points. Then the only solution $\varphi: \mathbb{R} \rightarrow \mathbb{R}$ of (7.3) such that $\varphi(1)=1$ is the identity function.

\section{Schröder's equation}

In this part we would like to present in details six results on solutions $\varphi$ of the Schröder equation

$$
\varphi(f(x))=\varrho \varphi(x) .
$$

The first concerns convex solutions and was proved by J. Walorski [258; §2]; see also [130] by A. Joffe and F. Spitzer.

Let $X$ be a Banach space with $\operatorname{dim} X \geq 1$ and let $K \subset X$ be a closed cone with int $K \neq \emptyset$ and such that $K \cap(-K)=\{0\}$. Define an order $\leq$ on $X$ by the formula

$$
x \leq y \quad \text { if and only if } \quad y-x \in K
$$

and assume that $\left.\|\cdot\|\right|_{K}$ is an increasing function. Further, let $A: X \rightarrow X$ be a continuous linear operator which maps bounded subsets of $X$ into relatively compact ones, satisfying

$$
A(K) \subset K
$$

and such that for every $x \in K \backslash\{0\}$ there exists a positive integer $n$ with $A^{n} x \in$ int $K$. By the Krein-Rutman theorem there exists exactly one $u \in \operatorname{int} K$ and exactly one continuous linear functional $g: X \rightarrow \mathbb{R}$ such that

$$
A u=\varrho u,
$$


Vol. 61 (2001)

$$
\begin{gathered}
g(A x)=\varrho g(x) \quad \text { for every } \quad x \in X, \\
g(x)>0 \quad \text { for } \quad x \in K \backslash\{0\}, \\
\|u\|=1 \quad \text { and } \quad g(u)=1,
\end{gathered}
$$

where $\varrho$ denotes the spectral radius of $A$ :

$$
\varrho=\lim _{n \rightarrow \infty}\left\|A^{n}\right\|^{1 / n}
$$

Consider also a function $f: K \rightarrow K$ such that

$$
\begin{gathered}
f(x) \neq 0 \quad \text { for every } \quad x \in K \backslash\{0\}, \\
\lim _{x \rightarrow 0} \frac{f(x)-A x}{\|x\|}=0, \\
g(x) \geq c\|g\|\|x\| \quad \text { for every } \quad x \in f(K)
\end{gathered}
$$

where $c$ is a positive real constant.

Theorem 8.1. Assume $f$ is increasing, convex and

$$
\lim _{n \rightarrow \infty} f^{n}(x)=0 \quad \text { for every } \quad x \in K \backslash\{0\} .
$$

If $\varrho<1$ and $a \in \operatorname{int} K$ then:

(i) For every $x \in K$ the sequence $\left(\frac{g\left(f^{n}(x)\right)}{g\left(f^{n}(a)\right)}: n \in \mathbb{N}\right)$ converges and the function $\varphi_{0}: K \rightarrow[0, \infty)$ defined by

$$
\varphi_{0}(x)=\lim _{n \rightarrow \infty} \frac{g\left(f^{n}(x)\right)}{g\left(f^{n}(a)\right)}
$$

is an increasing and convex solution of (8.1) and

$$
\varphi_{0}(x)=\lim _{n \rightarrow \infty} \frac{\left\|f^{n}(x)\right\|}{\left\|f^{n}(a)\right\|} \quad \text { for every } \quad x \in K .
$$

(ii) If $\varphi: K \rightarrow \mathbb{R}$ is an increasing and convex solution of (8.1) then

$$
\varphi(x)=\varphi(a) \varphi_{0}(x) \quad \text { for every } \quad x \in K .
$$

Regarding some further results obtained via the Krein-Rutman theorem see also [256] by J. Walorski. 
The second result deals with differentiable solutions of (8.1). Let $X$ be a Banach space, $r$ be a positive integer, $\delta \in[0, r], f$ be a diffeomorphism of the class $C^{r}$ defined on a neighbourhood of the origin of $X$ and taking values in $X$. Assume $f(0)=0$ and put

$$
\varrho=f^{\prime}(0) .
$$

Consider now (local) solutions $\varphi: U \rightarrow X$ of class $C^{r}$ of (8.1) such that

$$
\sup \left\{\frac{\left\|\varphi^{(r)}(x)-\varphi^{(r)}(0)\right\|}{\|x\|^{\delta}}: x \in U \backslash\{0\}\right\}<\infty
$$

and

$$
\varphi^{(k)}(0)=\varphi_{0}^{(k)}(0) \quad \text { for every } \quad k \in\{1, \ldots, r\},
$$

where $\varphi_{0}$ is a fixed mapping of class $C^{r}$ defined on a neighbourhood of the origin, taking values in $X$, and such that

$$
\left(\varphi_{0} \circ f-\varrho \circ \varphi_{0}\right)^{(k)}(0)=0 \quad \text { for every } \quad k \in\{1, \ldots, r\} .
$$

Following M. Sablik [222; Corollary 1.1] we can formulate the following.

Theorem 8.2. If

$$
\|\varrho\|^{r+\delta}\left\|\varrho^{-1}\right\|<1
$$

and there exists a positive real number $R$ such that

$$
\sup \left\{\frac{\left\|f^{(r)}(x)-f^{(r)}(0)\right\|}{\|x\|^{\delta}}: 0<\|x\| \leq R\right\}<\infty
$$

then in a neighbourhood $U$ of the origin equation (8.1) has exactly one solution $\varphi: U \rightarrow X$ of class $C^{r}$ satisfying (8.2) and (8.3).

In fact [222] by M. Sablik concerns differentiable solutions of much more general equations, viz. (1.2) and (1.1).

Regarding analytic solutions see the paper [240] by W. Smajdor where the classical Koenig's theorem has been extended to Banach spaces.

As well known the Schröder equation is strictly connected with the iteration theory. Here we only mention the papers [82] and [83] by J. Domsta dealing with regularly varying solutions as well as the following his result from [84].

Assume $f:(0, \infty) \rightarrow(0, \infty)$ is continuous, increasing and $f(x)<x$ for $x \in$ $(0, \infty)$. Let

$$
\varrho=\lim _{x \rightarrow 0} \frac{f(x)}{x}
$$


exists and belongs to the open interval $(0,1)$. Define $F:[0, \infty) \rightarrow(0,1)$ by

$$
F(x)= \begin{cases}\frac{f(x)}{x} & \text { for } \quad x \in(0, \infty), \\ \varrho & \text { for } \quad x=0 .\end{cases}
$$

Theorem 8.3. If either $F$ is of bounded variation in a neighbourhood of zero, or

$$
F(x)-\varrho=O\left(|\log x|^{-1-\nu}\right), \quad x \rightarrow 0,
$$

with a positive $\nu$, then for every $a \in(0, \infty)$ equation (8.1) has exactly one regularly varying solution $\varphi:(0, \infty) \rightarrow(0, \infty)$ such that $\varphi(a)=1$; it is given by the formula

$$
\varphi(x)=\lim _{n \rightarrow \infty} \frac{f^{n}(x)}{f^{n}(a)} .
$$

The second domain involving the Schröder equation is dynamics. Regarding the measurable case in pretty general spaces the reader is referred to the booklet [262] by B. Weiss. However, the classical example of appearance of Schröder's equation in dynamics is the analytic one. There are two new monographs and a remarkable paper devoted to this classical but more and more vivid domain: [29] by A. F. Beardon, [242] by N. Steinmetz, and [265] by J.-C. Yoccoz. What concerns not so recent works we call attention of the reader to the article [58] by A. D. Bryuno as well as to the paper [218] by H. Rüssmann and to the unnoticed, as it seems, booklet [259] by B. Ware and we mention the paper [272] by E. Zehnder. One of the leading topics of them is connected with the so-called Siegel set, i.e. the set $S$ of all $\varrho \in \mathbb{C}$ such that $|\varrho|=1$ and for every analytic in a neighbourhood of the origin function $f$ with $f(0)=0$ and $f^{\prime}(0)=\varrho$ equation (1) has a local analytic invertible solution. According to A. D. Bryuno [58; Theorem 6 on p. 226], see also [57], and [218] by H. Rüssmann, if the denominator $q_{n}(n \in \mathbb{N} \cup\{0\})$ of the $n$-th convergent in the regular continued fraction expansion of an irrational $\alpha$ satisfies

$$
\sum_{n=0}^{\infty} \frac{\log q_{n+1}}{q_{n}}<\infty
$$

then $\exp (2 \pi \mathrm{i} \alpha) \in S$; see also [242; Chapter 4, Section 2]. As follows from a theorem of J.-C. Yoccoz [265; p. 6], [264; p. 55], the Bryuno condition actually characterizes the Siegel set. Another characterization of the Siegel set may be seen from the main result of the second part of the Yoccoz' paper [265]; cf. [264; Remarque d].

Theorem 8.4. Let $\varrho \in \mathbb{C}$ satisfy $|\varrho|=1$. If the Schröder equation

$$
\varphi(\varrho x(1-x))=\varrho \varphi(x)
$$

has a local analytic invertible solution then equation (8.1) has a local analytic invertible solution for every analytic in a neighbourhood of the origin function $f$ with $f(0)=0$ and $f^{\prime}(0)=\varrho$. 
Regarding global properties of the Siegel set note that it is of the first Baire category subset of the unit circle (see [112; Remark on p. 610] by M. R. Herman) whereas, as it is well known due to C. L. Siegel, $S$ is of full Lebesgue measure. Some open problems concerning Siegel's theorem can be found in the survey paper [113] by M. R. Herman.

A reader interested in connections between Schröder's equation and operator theory is referred, for instance, to the paper [233] by J. H. Shapiro where the compactness problem for composition operators on Hardy spaces is mainly studied.

Many other fields of mathematics are known in which the Schröder equation is applicable. However, it is useful also outside of mathematics. As an example we quote the papers [2] and [3] by J. Aczél and M. Kuczma where the equation

$$
\varphi(k x)=k^{\gamma} \varphi(x)
$$

is studied to get its general solution and conditions under which the unique solution has the form $c x^{\gamma}$. This equation comes from meteorology. The knowledge how to solve another Schröder-type equation

$$
\varphi(a z)=b \varphi(z)
$$

is helpful in answering some questions appearing in the theory of neurons; results in this direction are listed in [1]. For more general case of equation

$$
\varphi\left(a_{1} z_{1}, \ldots, a_{N} z_{N}\right)=b \varphi\left(z_{1}, \ldots, z_{N}\right)
$$

see [17]. The reader can find there, for instance, what follows.

Theorem 8.5. Assume $a, b$ are complex numbers, $|a|=1$, and let $\varphi: \mathbb{C} \backslash\{0\} \rightarrow \mathbb{C}$ be a solution of (8.4) continuous on every circle around 0 .

(i) If $|b| \neq 1$ then $\varphi$ vanishes on $\mathbb{C} \backslash\{0\}$.

(ii) If $|b|=1$ and $\frac{\arg a}{\pi}$ is irrational then either $\varphi$ vanishes on $\mathbb{C} \backslash\{0\}$, or there exists an integer $k$ and $a$ function $\Phi:(0, \infty) \rightarrow \mathbb{C}$ such that $b=a^{k}$ and

$$
\varphi(z)=z^{k} \Phi(|z|) \quad \text { for every } \quad z \in \mathbb{C} \backslash\{0\} .
$$

What concerns completely monotonic solutions of (8.4) in the real case we have the following result by S. Dubuc [85; Theorem 8].

Theorem 8.6. Assume $0<b<1<a$ and let $\varphi:(0, \infty) \rightarrow(0, \infty)$ be a completely monotonic solution of (8.4). Then there is a Borel measure $\mu$ on $[1, a)$ such that

$$
\varphi(x)=\int_{[1, a)} \sum_{n=-\infty}^{\infty} b^{-n} \exp \left(-x a^{n} t\right) \mu(d t)
$$

for every $x \in(0, \infty)$.

The author shows also (Theorem 9) that if $\varphi$ is a completely monotonic solution of (8.4) then $x^{c} \varphi(x)$ with $c=-\frac{\log b}{\log a}$ is almost constant. 


\section{Abel's equation}

The problem of the existence and uniqueness of decreasing and convex solutions of the Abel equation

$$
\varphi(f(x))=\varphi(x)+1
$$

defined on cones in Banach spaces was examined by J. Walorski [257]. As in the case of Theorem 8.1 the main tool of the proof is the Krein-Rutman theorem.

The existence of continuous solutions of (9.1) strictly depends on the dynamics of $f$. To state a result of G. Belitskii and Yu. Lyubich [31; Corollary 1.6] consider a continuous self-mapping $f$ of a topological space $X$. A subset of $X$ is called wandering if it has a neighbourhood $U$ such that $\operatorname{cl} f^{m}(U) \cap \operatorname{cl} f^{n}(U)=\emptyset$ for all positive integers $m, n$ with $|m-n|$ large enough.

Theorem 9.1. Assume $X$ is locally compact and $\sigma$-compact. If $f: X \rightarrow X$ is continuous and injective then the following statements are equivalent.

(i) There exists a continuous solution $\varphi: X \rightarrow \mathbb{C}$ of (9.1).

(ii) For every continuous $G: X \rightarrow \mathbb{C} \backslash\{0\}$ and $H: X \rightarrow \mathbb{C}$ there exists a continuous solution $\varphi: X \rightarrow \mathbb{C}$ of (1.3).

(iii) Every compact subset of $X$ is wandering.

Related problems for analytic solutions on manifolds and Lie groups are studied in [32].

All the homeomorphic solutions as well as diffeomorphic ones with positive Jacobian on the plane of the Abel equation have been described by Z. Leśniak [163].

Studying iterations of $1-\mathrm{e}^{-z}$ and $\log (1+z)$ at zero P. L. Walker [255] constructed two distinct solutions of

$$
\varphi\left(\exp \left(\frac{z}{\mathrm{e}}\right)\right)=\varphi(z)+1
$$

analytic on domains which include the intervals $(-\infty, \mathrm{e})$ and $(\mathrm{e}, \infty)$, respectively. Actually, the first one is analytic and satisfies (9.2) in particular for $\operatorname{Re} z<\mathrm{e}$ and has the period $2 \pi$ ie, whereas the second one is analytic on $\mathbb{C} \backslash(-\infty, \mathrm{e}]$ and satisfies (9.2) for $z \in \mathbb{C} \backslash(-\infty, \mathrm{e}]$ such that $|\operatorname{Im} z|<\pi$ e.

If $\varphi$ is an invertible solution of (9.1) then its inverse is a solution of the equation

$$
f(\varphi(z))=\varphi(z+1) .
$$

An algorithm of obtaining entire solutions of this equation related to (9.1) has been given by P. L. Walker [253; Theorem 2].

Theorem 9.2. Let $f$ be an entire function with $f(0)=0, f^{\prime}(0)=1, f^{\prime \prime}(0)>0$ and $f^{(n)}(0) \geq 0$ for every integer $n \geq 3$ and put

$$
a=\frac{1}{2} f^{(2)}(0), \quad b=\frac{1}{a}\left(\frac{f^{(3)}(0)}{6}-a^{2}\right) .
$$


If either $b \neq 0$, or

$$
f^{(4)}(0)<24 a^{3}
$$

then for every $z \in \mathbb{C}$ there exists the limit

$$
\lim _{n \rightarrow \infty} f^{n}\left(\frac{1}{a(n-z)+b \log n}\right)
$$

and defines an entire non-constant solution $\varphi$ of (9.3).

In the special case of $f(z)=\mathrm{e}^{z}-1$ some properties of this solution are derived in [254] by the same author. In particular, he locates its zeros and asymptotic values, shows that its derivative is never zero and for any $B>0$ the set $\{z \in$ $\mathbb{C}: \operatorname{Re} \varphi(z)>B\}$ has finite area (and, consequently, for any $A>2$ the set $\{z \in \mathbb{C}:|f(z)|>A\}$ has finite area).

The Abel equation in the form (9.3) appears also in studying the so-called Hardy fields, i.e. subfields of the ring of germs of continuous real-valued functions defined on $(0, \infty)$, closed under differentiation. This is the main subject of [52] by M. Boshernitzan where (9.3) with $f(x)=\mathrm{e}^{x}$ and $f(x)=\mathrm{e}^{x}-1$ is investigated in the class of germs.

Regarding applications of the Abel (as well as the Schröder) equation to differential equations the reader is referred to the book [196] by F. Neuman.

In examining simultaneous Abel equations

$$
\varphi\left(f_{1}(x)\right)=\varphi(x)+\lambda_{1}, \quad \varphi\left(f_{2}(x)\right)=\varphi(x)+\lambda_{2},
$$

the set $L$ of all limit points of the set

$$
\left\{f_{1}^{i} \circ f_{2}^{j}(x): i, j \in \mathbb{Z}\right\}
$$

plays a fundamental role. To be more precise fix a real open interval $I$ and a family $\mathcal{F}$ of commuting homeomorphisms of $I$ onto itself without fixed points. Fix $f_{1} \in \mathcal{F}$ and for $f \in \mathcal{F}$ denote by $\nu(f)$ the rotation number of the pair $\left(f_{1}, f\right)$.

Theorem 9.3. Assume $\mathcal{F}$ contains an $f_{2}$ such that for every $x \in I$ and for all $i, j \in \mathbb{Z} \backslash\{0\}$ we have $f_{2}^{j}(x) \neq f_{1}^{i}(x)$. Then:

(i) The set $L$ of all limit points of (9.4) does not depend on $x \in I$; moreover, either $L$ is a perfect nowhere dense set containing the endpoints of $I$, or $I \subset L$.

(ii) The system

$$
\varphi(f(x))=\varphi(x)+\nu(f), \quad f \in \mathcal{F},
$$

has a unique (up to an additive constant) continuous solution $\varphi: I \rightarrow \mathbb{R}$; this solution is monotonic, $\varphi(L \cap I)=\mathbb{R}$, and it is invertible if and only if $I \subset L$. 
Part (i) was proved by M. C. Zdun [268; Theorem 1]. Part (ii) comes from [128]. It generalizes [269; Theorem 2] by M. C. Zdun who studied the case of $\mathcal{F}$ consisting of two elements. His result shows also that if a system (9.5) with some $\nu$ has a continuous solution, then, in fact, $\nu$ is the rotation number up to a multiplicative constant.

In the case where for every $f \in \mathcal{F}$ and for every $x \in I$ there are $i, j \in \mathbb{Z} \backslash\{0\}$ such that $f^{j}(x)=f_{1}^{i}(x)$ results depend on the additive subgroup $G$ of $\mathbb{R}$ generated by $\nu(\mathcal{F})$. Namely, if $G$ is discrete then system (9.5) has a homeomorphic solution depending on an arbitrary function. If $G$ is dense then the situation is similar to that of Theorem 9.3 [128; Theorem 3]; see also [269; Theorem 1].

As follows from [103] by K. Gościcka the case of a family $\mathcal{F}$ consisting of functions not being surjective can be reduced to that one of homeomorphisms. Diffeomorphic solutions of (9.5) were studied by M. C. Zdun in [270]. Regarding two simultaneous Abel's equations on the plane see [165] by Z. Leśniak. An abstract case is treated in [223] by M. Sablik.

What concerns systems of two simultaneous Abel's inequalities the reader is referred to [164] by Z. Leśniak. It turns out that some of them are equivalent with the associated systems of equations.

\section{Miscellaneous results and problems}

In this section we are going to present some facts of individual character.

In the complex domain the problem of the convergence of formal solutions is still alive. The following result due to J.-P. Bézivin [41] serves as an example.

Theorem 10.1. Let $q$ and the coefficients of a non-zero polynomial $P$ be complex algebraic numbers. Assume that $|q|=1$ and $q$ is not a root of unity. If $\varphi$ is a formal solution of

$$
P(z, \varphi(z), \varphi(q z))=0
$$

and has algebraic coefficients then $\varphi$ has a positive radius of convergence.

Concerning a similar problem for other equations and fields see [44] by J.-P. Bézivin and A. Boutabaa and [42] and [43] by J.-P. Bézivin.

A straightforward computation shows that for every $a, b \in \mathbb{C}$ the function $\varphi$ defined in the open unit disc by

$$
\varphi(z)=a+\frac{b z}{1-z}
$$

is a solution of

$$
3 z \varphi\left(z^{3}\right)=3 z \varphi\left(z^{6}\right)+\varphi\left(z^{2}\right)+\lambda \varphi\left(\lambda z^{2}\right)+\lambda^{2} \varphi\left(\lambda^{2} z^{2}\right),
$$


where $\lambda=\mathrm{e}^{2 \pi \mathrm{i} / 3}$. It is a conjecture of L. Berg and G. Meinardus [37; Conjecture 3], [38; Conjecture 3] that functions (10.1) are the only analytic solutions of (10.2) in the open unit disc. It is interesting that this conjecture is equivalent to the positive answer to the Collatz " $3 n+1$ problem" [37], [38]. (Regarding $3 n+1$ problem and, in particular, some its history consult [263] by J. G. Wirsching.)

Beside those that describe functions which are additive on their graphs there are also other equations originating from the classical one of Cauchy:

$$
\begin{gathered}
\varphi(x+f(x))=\varphi(x)+\varphi(f(x)), \\
\varphi(f(x)+g(x))=\varphi(f(x))+\varphi(g(x)) .
\end{gathered}
$$

The first one expresses additivity of $\varphi$ on the graph of a given function $f$ and for the first time was studied by M. C. Zdun [267]. This equation appears naturally in some problems, cf. [177] by J. Matkowski and M. Sablik, [250] by M. S. Tomás, [118]. However, it is studied mainly because of its own interest. A typical result states that if $f: \mathbb{R} \rightarrow \mathbb{R}$ is continuous, $f(0)=0$ and $x f(x)>0$ for $x \in \mathbb{R} \backslash\{0\}$ then the only solution $\varphi: \mathbb{R} \rightarrow \mathbb{R}$ of (10.3) continuous in a neighbourhood of zero and differentiable at zero is of the form $c x$ ([88; Theorem 3] by G. L. Forti); see also [89] by G. L. Forti and [220] by M. Sablik for some generalizations. Equation (10.3) for real involutions $f$ is considered in [177] by J. Matkowski and M. Sablik and [61] by B. Choczewski, I. Corovei and C. Rusu, whereas for $f(x)=1 / x$ in [147] by P. Kostyrko; cf. also [61]. In the complex case equation (10.3) is studied in [50] by C. Borelli Forti and G. L. Forti. Some results concerning equation (10.4) can be found in [49] by C. Borelli Forti and [221] by M. Sablik. Other equations, e.g. Jensen's one, are considered on curves as well (see e.g. [93] and its extension [95] by J. Ger and M. Sablik, [119; Chapter II]).

We note here an interesting phenomenon known up to now mainly in the theory of the Cauchy equation, namely solutions with big graphs [151; Chapter XII, Section 4]. The paper [133] by P. Kahlig and J. Smítal seems to be the first one which deals with such solutions of iterative equations, namely those with superpositions of the unknown function. Being inspired by this paper L. Bartłomiejczyk has studied such solutions for a number of equations in [24]-[28]. The general results obtained there were applied by him to some special equations, in particular to that of Daróczy (cf. Section 6) and Schilling.

The following problem concerning the linear equation

$$
\varphi(f(x))=g(x) \varphi(x)+h(x)
$$

was posed by L. Reich [213]. He asked under what possibly weak assumptions on the given functions $f, g, h$ we can "embed" (10.5) in the equation with "continuous time" argument:

$$
\varphi(F(t, x))=G(t, x) \varphi(x)+H(t, x) .
$$


More precisely: under what assumptions on $f, g, h$ there exist $F, G, H$ from a suitable class of functions solving equations

$$
\begin{gathered}
F(t+s, x)=F(t, F(s, x)), \\
G(t+s, x)=G(s, x) G(t, F(s, x)), \\
H(t+s, x)=G(t, F(s, x)) H(s, x)+H(t, F(s, x))
\end{gathered}
$$

and fulfilling conditions

$$
F(1, \cdot)=f, \quad G(0, \cdot)=1, \quad G(1, \cdot)=g, \quad H(0, \cdot)=0, \quad H(1, \cdot)=h
$$

such that every solution of (10.5) from the considered function class satisfies equation (10.6). Up to now answers in the following cases are known: in the general one where none regularity of $\varphi, f, g, h$ and of $F, G, H$ is required which was studied by Z. Moszner [194], in the class of formal power series due to L. Reich (oral communication) and in the class of continuous functions on a real interval where an answer was given by G. Guzik [109].

Up to now we have considered only single-valued solutions. What concerns set-valued solutions we quote here a result of A. Smajdor and W. Smajdor [239; Theorem 4] which shows that if the functions are single-valued then, in general, minimal solutions are almost everywhere single-valued.

Theorem 10.2. Assume $X$ is a metric space, $f: X \rightarrow X$ is continuous and open whereas $h: X \times \mathbb{R} \rightarrow \mathbb{R}$ is continuous and increasing in the second variable. Let $\varphi_{0}$ be an upper semi-continuous set-valued function defined on $X$, with compact interval values which are contained in a compact set of reals and such that

$$
\varphi_{0}(x) \supset h\left(x, \varphi_{0}[f(x)]\right) \quad \text { for } \quad x \in X .
$$

Then there exists a minimal (in the sense of inclusion) upper semi-continuous set-valued function $\varphi$ defined on $X$, with compact interval values $\varphi(x) \subset \varphi_{0}(x)$ satisfying

$$
\varphi(x)=h(x, \varphi[f(x)]) \quad \text { for } \quad x \in X,
$$

and except a set of the first category the values $\varphi(x)$ of each such a minimal solution are singletons.

For some related results consult [241] by W. Smajdor.

Although, as it seems, general solutions are not very important in the theory of iterative functional equations, there are some interesting results in this direction. The following, originating from one of the last paper by M. Kuczma [152; Proposition 3], can serve as an example. It concerns the equation

$$
\varphi(z) \overline{\varphi(-1 / \bar{z})}=-1
$$


which comes from astrophysics.

Theorem 10.3. The general solution $\varphi: \mathbb{C} \backslash\{0\} \rightarrow \mathbb{C} \backslash\{0\}$ of (10.7) is given by

$$
\varphi(z)=z f(z) / \overline{f(-1 / \bar{z})}
$$

where $f: \mathbb{C} \backslash\{0\} \rightarrow \mathbb{C} \backslash\{0\}$ is an arbitrary function.

Taking $f(z)=c z^{n}$ in (10.8) where $c \neq 0$ is a constant and $n$ is an integer we obtain the solution

$$
\varphi(z)=\eta z^{2 n+1}
$$

with $\eta$ from the unit circle. According to [152; Theorem] it is the unique solution of (10.7) which is analytic on $\mathbb{C} \backslash\{0\}$ and has a removable singularity or a pole at the origin.

Except Abel's and Schröder's equations we do not pay attention to a very important and still vivid topic of conjugation which lies, no doubt, in the theory of iterative functional equations. However, we would like to remaind the following problem posed by Gy. Targonski [248]: Characterize all self-mappings of the plane which are conjugated to a complex polynomial (a) under a diffeomorphism, (b) under a homeomorphism, (c) under a bijection.

We finish with calling attention to a very interesting (more than thirty years old) problem posed by Z. Moszner [192]. To state it fix $r \in \mathbb{N} \cup\{\infty\}$ and $k \in \mathbb{N}$ and denote

$D_{k}^{r}=\left\{f: \mathbb{R}^{k} \rightarrow \mathbb{R}^{k}: f\right.$ is of the class $C^{r}$ and $\operatorname{Det} f^{\prime}(x)>0$ for every $\left.x \in \mathbb{R}^{k}\right\}$.

The problem reads as follows. Let $f \in D_{k}^{r}$. Does there exist an $n \in \mathbb{N}$ and $\varphi_{1}, \ldots, \varphi_{n} \in D_{k}^{r}$ such that

$$
f=\varphi_{1}^{2} \circ \ldots \circ \varphi_{n}^{2} .
$$

For $k=1$ this problem was solved (positively, $n=4$ is enough) by M. Kuczma [149]. For $k>1$ it is still open.

Acknowledgement. This research was supported by the Silesian University Mathematics Department (Iterative Functional Equations program).

\section{References}

[1] J. ACZÉL, Results on a 'folk equation', Numbers, Functions, Equations '98 (Noszvaj, 1998), 57-59, Leaflets in Mathematics, Pécs, 1998.

[2] J. ACZÉL AND M. Kuczma, Generalizations of a "folk-theorem" on simple functional equations in a single variable, Results Math. 19 (1991), 5-21. MR 92d:39017

[3] J. ACZÉL AND M. KuCZMA, Solutions of a functional equation, convex of higher order, General inequalities 6 (Oberwolfach, 1990), 209-213, Internat. Ser. Numer. Math. 103, Birkhäuser, Basel, 1992. MR 94d:39007 
[4] C. Alsina, On some iterative functional equations involving $t$-norms, European Conference on Iteration Theory (Caldes de Malavella, 1987), 408-412, World Sci. Publishing, Teaneck, 1989. MR 92c:39013

[5] J. Appell AND P. P. Zabrejko, Nonlinear superposition operators, Cambridge Tracts in Mathematics 95, Cambridge University Press, Cambridge, 1990. MR 91k:47168

[6] N. Aronszajn, Differentiability of Lipschitzian mappings between Banach spaces, Studia Math. 57 (1976), 147-190. MR 54\#13562

[7] Yu. V. Azarina, Invariant analytic curves for entire functions [Russian], Sibirsk. Mat. Zh. 30 (1989), 3-8; English translation: Siberian Math. J. 30 (1989), 349-353. MR 90j:30038

[8] M. BADIALE, Some characterization of the q-gamma function by functional equations. I, Atti Accad. Naz. Lincei Rend. Cl. Sci. Fis. Mat. Natur. (8) 74 (1983), 7-11; II, ibidem 49-54. MR 86b:33001a,b

[9] J. A. BAKER, Functional equations, tempered distributions and Fourier transforms, Trans. Amer. Math. Soc. 315 (1989), 57-68. MR 90k:39006

[10] J. A. BAKER, A functional equation from probability theory, Proc. Amer. Math. Soc. 121 (1994), 767-773. MR 94i:39006

[11] J. A. BAKER, Functional equations and Weierstrass transforms, Results Math. 26 (1994), 199-204. MR 96a:39025

[12] J. A. BAKER, Mean value theorems via spectral synthesis, J. Math. Anal. Appl. 193 (1995), 306-317. MR 96k:39028

[13] K. BARon, A remark on linear functional equations in the indeterminate case, Glas. Mat. Ser. III 20(40) (1985), 373-376. MR 87g:39015

[14] K. Baron, Remark P283R2, Aequationes Math. 35 (1988), 301-303.

[15] K. BARon, On a problem of R. Schilling, Selected topics in functional equations (Graz, 1986), Ber. No. 286, Ber. Math.- Statist. Sekt. Forschungsgesellsch. Joanneum, Forschungszentrum Graz, Graz, 1988. MR 90g:39013

[16] K. Baron, On a problem of Z. Daróczy, Zeszyty Nauk. Politech. Śląsk. Mat.-Fiz. 64 (1990), $51-54$.

[17] K. Baron, Appendix to the chapter Synthesis of the firing of many neurons of The brain: Unraveling the mystery of how it works by T. L. Saaty, RWS Publications, Pittsburgh, 2000; 339-347.

[18] K. BARON And W. JARCZYK, On a way of division of segments, Aequationes Math. 34 (1987), 195-205. MR 89b:39003

[19] K. BARON AND W. JARCZYK, Random-valued functions and iterative functional equations, manuscript.

[20] K. BARON AND A. Simon, On approximate solutions of an iterative functional equation, Ann. Math. Sil. 8 (1994), 79-84. MR 96e:39018

[21] K. Baron, A. Simon And P. Volkmann, Solutions d'une équation fonctionnelle dans l'espace des distributions tempérées, C. R. Acad. Sci. Paris. Ser. I Math. 319 (1994), 12491252. MR 96b:39023

[22] K. Baron and P. Volkmann, Unicité pour une équation fonctionnelle, Rocznik Nauk.Dydakt. Prace Mat. 13 (1993), 53-56. MR 96j:39026

[23] L. BartŁomiejczyk, Some remarks on the Daróczy equation, Ann. Math. Sil. 9 (1995), 47-63. MR 97b:39039

[24] L. BArtŁomiejczyK, Solutions with big graph of homogeneous functional equations in a single variable, Aequationes Math. 56 (1998), 149-156. MR 99f:39032

[25] L. BARTŁOMIEJCZYK, Solutions with big graph of iterative functional equations of the first order, Colloq. Math. 82 (1999), 223-230. MR 2000m:39052

[26] L. BArtŁomiejczyK, Solutions with big graph of an equation of the second iteration, Aequationes Math. to appear.

[27] L. BartŁomiejczyK, Solutions with big graph of the equation of invariant curves, Bull. Polish Acad. Sci. Math.

[28] L. BARTŁOMIEJCZYK, Iterative roots with big graph, Real Anal. Exchange.

[29] A. F. BEARDon, Iteration of rational functions. Complex analytic dynamical systems, Graduate Text in Mathematics 132, Springer-Verlag, New York, 1991. MR 92j:30026 
[30] G. BeLITSKII, Multidimensional functional equations generated by affine transformations, Oper. Theory Adv. Appl. 98 (1997), 41-54. MR 98j:47067

[31] G. Belitskil And Yu. Lyubich, The Abel equation and total solvability of linear functional equations, Studia Math. 127 (1998), 81-97. MR 99e:39081

[32] G. Belitskil And Yu. LyUBich, The real-analytic solutions of the Abel functional equation, Studia Math. 134 (1999), 135-141. MR 2000f:39022

[33] G. Belitskil And V. Nicolaevsky, Linear functional equations on the line, Integral Equations Operator Theory 21 (1995), 212-223. MR 96d:39006

[34] G. Belitskit and V. TKaChenKo, Locally analytic solvability of multidimensional functional equations in a neighbourhood of a nonsingular point [Russian], Dokl. Akad. Nauk. SSSR 314 (1990), 215-217; English translation: Soviet Math. Dokl. 42 (1991), 239-242. MR 92g:39006

[35] G. Belitskit And V. TKachenko, Analytic solvability of multidimensional functional equations in a neighbourhood of a nonsingular point [Russian], Teor. Funktsiř Funktsional. Anal. i Prilozhen. 58 (1992), 7-21. MR 95d:39008

[36] G. Belitskil and V. TKaChenko, On solvability of linear difference equations in smooth and real analytic vector functions of several variables, Integral Equations Operator Theory 18 (1994), 123-129. MR 95d:39001

[37] L. Berg And G. Meinardus, Functional equations connected with the Collatz problem, Results Math. 25 (1994), 1-12. MR 95d:11025

[38] L. Berg And G. Meinardus, The $3 n+1$ Collatz problem and functional equations, Rostock. Math. Kolloq 48 (1995), 11-18. MR 97e:11034

[39] D. Bessis, S. Marmi and G. Turchetti, On the singularities of divergent majorant series arising from normal form theory, Rend. Math. Appl. (7) 9 (1989), 645-659. MR 91e:58162

[40] J.-P. BÉZivin, Sur les équations fonctionnelles aux itérées, Aequationes Math. 39 (1990), 68-77. MR 91c:39008

[41] J.-P. BÉZIVIn, Convergence des solutions formelles de certaines équations fonctionnelles, Aequationes Math. 44 (1992), 84-99. MR 93d:39011

[42] J.-P. BÉZIvin, Sur une classe d'équations fonctionnelle non linéaires, Funkcial. Ekvac. 37 (1994), 263-271. MR 96a:39021

[43] J.-P. BÉZIVIn, Solutions rationnelles de certaines équations fonctionnelles, Aequationes Math. 36 (1998), 112-124. MR 90c:11015

[44] J.-P. BÉZIVIn AND A. BoutaBAA, Sur les équations fonctionnelles p-adiques aux q-différences, Collect. Math. 43 (1992), 125-140. MR 95j:39031

[45] J.-P. BÉZIVIN AND F. GRAmaIn, Solutions entières d'un système d'équations aux différences, Ann. Inst. Fourier (Grenoble) 43 (1993), 791-814. MR 95e:30026

[46] A. Blokh, E. Coven, M. Misiurewicz and Z. Nitecki, Roots of continuous piecewise monotone maps of an interval, Acta Math. Univ. Comenian. (N.S.) 60 (1991), 3-10. MR 92i:58086

[47] A. M. BlokH, The set of all iterates is nowhere dense in $C([0,1],[0,1])$, Trans. Amer. Math. Soc. 333 (1992), 787-798. MR 93c:26003

[48] S. Bogatyi, On the nonexistence of iterative roots, Topology Appl. 76 (1997), 97-123. MR 98c:58135

[49] C. Borelli Forti, Redundancy conditions for the functional equation $f(k(t)+h(t))=$ $f(k(t))+f(h(t))$ [Italian], Stochastica 11 (1987), 93-105. MR 90g:39014

[50] C. Borelli Forti and G. L. Forti, On entire functions additive on graphs, C. R. Math. Rep. Acad. Sci. Canada 11 (1989), 29-33. MR 90h:30063

[51] J. M. Borwein AND R. Girgensohn, Functional equations and distribution functions, Results Math. 26 (1994), 229-237. MR 96b:39019

[52] M. Boshernitzan, Hardy fields and existence of transexponential functions, Aequationes Math. 30 (1986), 258-280. MR 88b:26003

[53] A. BötTCher AND H. HeIdLeR, On linear functional equations with two involutions, Seminar Analysis (Berlin, 1989/1990), 31-43, Karl-Weierstrass-Inst. Math., Berlin, 1990. MR 92h:39011 
[54] A. BoutabaA, Solutions entières p-adiques d'une certaine équation fonctionnelle, Séminaire d'Analyse, 1992-1993 (Albière), Sémin. Anal. Univ. Blaise Pascal (Clermont II) 8, Univ. Blaise Pascal (Clermont II), Clermont-Ferrand, 1996. MR 99a:11085

[55] A. BoutabaA, On some p-adic functional equation, $p$-adic functional analysis (Nijmegen, 1996), 49-59, Lecture Notes in Pure and Appl. Math. 192, Dekker, New York, 1997. MR 98g:12011

[56] E. M. BRonshteǏn, On an iterative square root of a quadratic trinomial [Russian], Geometric problems in the theory of functions and sets [Russian], 24-27, Kalinin. Gos. Univ., Kalinin, 1989. MR 93d:26003

[57] A. D. BRyuno, On convergence of transforms of differential equations to the normal form [Russian], Dokl. Akad. Nauk SSSR 165 (1965), 987-989. MR 33\#325

[58] A. D. Bryuno, Analytic form of differential equations [Russian], Tr. Mosk. Mat. Obs. 25 (1971), 119-262; II. Tr. Mosk. Mat. Obs. 26 (1972), 199-239; English translation: Trans. Moscow Math. Soc. 25 (1971), 131-288 (1973); II Trans. Moscow Math. Soc. 26 (1972), 199-239 (1974). MR 51\#13365

[59] R. Cheng, A. Dasgupta, B. R. Ebanks, L. F. Kinch, L. M. Larson and R. B. McFadDEN, When does $f^{-1}=1 / f$ ?, Amer. Math. Monthly 105 (1998), 704-717. MR 99h:39026

[60] B. Choczewski, Problem 27, Report of meeting. The Twenty-eight International Symposium on Functional Equations (Graz-Mariatrost, 1990), Aequationes Math. 41 (1991), 296.

[61] B. Choczewski, I. Corovei And C. Rusu, Cauchy's equation on the graph of an involution, Math. Pannon. 5 (1994), 197-202. MR 95i:39012

[62] B. Choczewski And M. Kuczma, On iterative roots of polynomials, European Conference on Iteration Theory (Lisbon, 1991), 59-67, World Sci. Publishing, Singapore, 1992.

[63] G. Choquet and J. Deny, Sur l'équation de convolution $\mu=\mu * \sigma$, C. R. Acad. Sci. Paris 250 (1960), 799-801. MR 22\#9808

[64] J. P. R. Christensen, Topology and Borel structure. Descriptive topology and set theory with applications to functional analysis and measure theory, North-Holland Mathematical Studies 10, North-Holland Publishing Co., Amsterdam-London; American Elsevier Publishing Co., Inc., New York, 1974. MR 50\#1221

[65] J. P. R. Christensen And P. Fischer, Linear independence of iterates and entire solutions of functional equations, Proc. Amer. Math. Soc. 103 (1988), 1120-1124. MR 89m:30051

[66] J. P. R. Christensen And P. Fischer, Linear independence of iterates and meromorphic solutions of functional equations, Proc. Amer. Math. Soc. 120 (1994), 1137-1143. MR 94f:30034

[67] R.-L. Clerc And C. Hartmann, Invariant curves as solutions of functional equations, Functional equations: history, applications and theory 217-226, Math. Appl., Reidel, Dordrecht-Boston, 1984. MR 89g:58104

[68] D. Colella And CH. Heil, Characterizations of scaling functions: continuous solutions, SIAM J. Matrix Anal. Appl. 15 (1994), 496-518. MR 95f:26004

[69] J.-P. ConZe AND A. RAUGI, Fonctions harmoniques pour un opérateur de transition et applications, Bull. Soc. Math. France 118 (1990), 273-310. MR 92g:60100

[70] M. CsÖRnYEI, Aronszajn null and Gaussian null sets coincide, Israel J. Math. 111 (1999), 191-201. MR 2000f:46057

[71] K. DANKIEWICZ, On approximate solutions of a functional equation in the class of differentiable functions, Ann. Polon. Math. 49 (1989), 247-252. MR 90f:39015

[72] Z. DARóczy, Problem P283, Aequationes Math. 32 (1987), 136-137.

[73] W. F. Darsow, M. J. Frank And H.-H. Kairies, Functional equations for a function of van der Waerden type, Rad. Mat. 4 (1988), 361-374; Errata: Rad. Mat. 5 (1989), 179-180. MR 90h:39015a,b

[74] I. Daubechies AND J. C. LAGarias, Two-scale difference equations. I. Existence and global regularity of solutions, SIAM J. Math. Anal. 22 (1991), 1388-1410; II. Local regularity, infinite products of matrices and fractals, SIAM J. Math. Anal. 23 (1992), 1031-1079. MR 92d:39001, 93g:39001 
[75] A. Deliu And M. C. Spruill, Existence results for refinement equations, Aequationes Math. 59 (2000), 20-37.

[76] J. Deny, Sur l'équation de convolution, Séminaire de Théorie du Potentiel: 4e Année 1959/60. Secretariat Math., Paris, 1961. Zbl. 101, 319

[77] G. DERfEL, The behaviour of solutions of functional and functional differential equations with certain transformations of the argument [Russian], Ukrain. Mat. Zh. 34 (1982), 350356; English translation: Ukrainian Math. J. 34 (1982), 286-291. MR 83i:34087

[78] G. DERFEL, A probabilistic method for studying a class of functional-differential equations [Russian], Ukrain. Math. J. 41 (1989), 1322-1327; English translation: Ukrainian Math. J. 41 (1989), 1137-1141. MR 90j:34095

[79] G. Derfel, N. Dyn AND D. Levin, Generalized refinement equations and subdivision processes, J. Approx. Theory 80 (1995), 272-297. MR 95k:45003

[80] G. Derfel AND R. Schilling, Spatially chaotic configurations and functional equations with rescaling, J. Phys. A 29 (1996), 4537-4547. MR 97i:39012

[81] J. Dhombres, Some aspects of functional equations, Lecture Notes. Chulalongkorn University, Department of Mathematics, Bangkok, 1979. MR 80j:39001

[82] J. Domsta, Regularly varying solutions of the Schröder equation, Uniwersytet Gdański, Preprint No 100, July 1994.

[83] J. Domsta, Regular iteration groups parametrized by dense sets of reals, Uniwersytet Gdański, Preprint No 101, August 1994.

[84] J. Domsta, Regularly varying solutions of Schröder's and related linear equations, manuscript.

[85] S. Dubuc, An approximation of the gamma function, J. Math. Anal. Appl. 146 (1990), 461-468. MR 91e:65031

[86] K. B. Dunn And R. LidL, Iterative roots of functions over finite fields, Math. Nachr. 115 (1984), 319-329. MR 85k:39006

[87] W. Förg-RoB, On a problem of R. Schilling I, Math. Pannon. 5 (1994), 29-65; II. ibidem 145-168. MR 95g:39024, 96e:39012

[88] G. L. ForTi, On some conditional Cauchy equations on thin sets, Boll. Un. Mat. Ital. B (6) 2 (1983), 391-402. MR 84h:39007

[89] G. L. FORTI, Redundancy conditions for the functional equation $f(x+h(x))=f(x)+$ $f(h(x))$, Z. Anal. Anwendungen 3 (1984), 549-554. MR 86c:39010

[90] B. GawEe, On the uniqueness of continuous solutions of functional equations, Ann. Polon. Math. 60 (1995), 231-239. MR 96b:39027

[91] B. GAwEe, Some functional inequalities and their Baire category properties, Aequationes Math. 52 (1996), 55-71. MR 98m:39084

[92] B. GAWEe, Linear functional equations and their Baire category properties, Aequationes Math. 60 (2000), 315-320.

[93] J. Ger And M. Sablik, On Jensen equation on a graph, Zeszyty Nauk. Politech. Śląsk. Mat.-Fiz. 68 (1993), 41-51.

[94] J. Ger And M. SABlik, The law of large numbers and a functional equation, Ann. Polon. Math. 68 (1998), 165-175. MR 99b:39026

[95] J. GER AND M. SABLIK, On a functional equation connected with the Jensen equation, European Conference on Iteration Theory (Urbino, 1996). Grazer Math. Ber. 339 (1999), $181-188$.

[96] R. Girgensohn, Funktionalgleichungen für nirgends differenzierbare Funktionen, Ph. D. thesis, Technische Universität Clausthal, Clausthal, 1992.

[97] R. Girgensohn, Functional equations and nowhere differentiable functions, Aequationes Math. 46 (1993), 243-256. MR 94j:39013

[98] R. Girgensohn, Nowhere differentiable solutions of a system of functional equations, Aequationes Math. 47 (1994), 89-99. MR 94k:39063

[99] R. Girgensohn, Remark 17, Report of meeting. The Thirty-second International Symposium on Functional Equations (Gargnano, 1994). Aequationes Math. 49 (1995), 190. 
[100] R. Girgensohn, Constructing singular functions via Farey fractions, J. Math. Anal. Appl. 203 (1996), 127-141. MR 97f:26006

[101] R. Girgensohn and J. Morawiec, Positivity of Schilling functions, Bull. Polish. Acad. Sci. Math. 48 (2000), 407-412.

[102] L. B. GonzÁLEZ, Linear independence of iterates of entire functions, Proc. Amer. Math. Soc. 112 (1991), 1033-1036. MR 91j:30020

[103] K. GościckA, On commutable functions and Abel's equations, Opuscula Math. 6 (1990), 59-76. MR 92f:39012

[104] F. Gramain, Équations aux différences et polynômes exponentiels, C. R. Acad. Sci. Paris Ser. I Math. 313 (1991), 131-134. MR 92h:30057

[105] M. GRINČ, On non-negative measurable solutions of a difference functional equation, Positivity 2 (1998), 221-228. MR 2000f:39024

[106] D. Gronau and J. Matkowski, Geometrical convexity and generalizations of the BohrMollerup theorem on the gamma function, Math. Pannon. 4 (1993), 153-160. MR 95a:33003

[107] D. Gronau and J. MatKowski, Geometrically convex solutions of certain difference equations and generalized Bohr-Mollerup type theorems, Results Math. 26 (1994), 290-297. MR 95k:39020

[108] D. Gronau And M. Sablik, A functional equation arising from an asymptotic formula for iterates, Ann. Math. Sil. 8 (1994), 173-187. MR 96f:39015

[109] G. GUZIK, On embedding of a linear functional equation, Rocznik Nauk.-Dydakt. Prace Mat. 16 (1999), 23-33.

[110] H. Haruki and Th. M. Rassias, New integral representations for Bernoulli and Euler polynomials, J. Math. Anal. Appl. 175 (1993), 81-90. MR 94e:39016

[111] CH. HeIl and D. Colella, Dilation equations and the smoothness of compactly supported wavelets, Wavelets: mathematics and applications, 163-201, Stud. Adv. Math., CRC, Boca Raton, 1994. MR 94j:42049

[112] M. R. HeRman, Are there critical points on the boundaries of singular domains?, Comm. Math. Phys. 99 (1985), 593-612. MR 86j:58067

[113] M. R. HeRman, Recent results and some open questions on Siegel's linearization theorem of germs of complex analytic diffeomorphisms of $C^{n}$ near a fixed point, VIIIth international congress on mathematical physics (Marseille, 1986), 138-184, World Sci. Publishing, Singapore, 1987. MR 89k:32013

[114] P. D. Humke And M. LaCzKovich, The Borel structure of iterates of continuous functions, Proc. Edinburgh Math. Soc. (2) 32 (1989), 483-494. MR 90i:28004

[115] B. R. Hunt, T. Sauer and J. A. Yorke, Prevalence: A translation-invariant "almost every" on infinite dimensional spaces, Bull. Amer. Math. Soc. (N.S.) 27 (1992), 217-238; Addendum: Bull. Amer. Math. Soc. (N.S.) 28 (1993), 306-307. MR 93k:28018, 93k:28019

[116] W. JARCZYK, Nonlinear functional equations and their Baire category properties, Aequationes Math. 31 (1986), 81-100. MR 87m:39007

[117] W. JARCZYK, On continuous functions which are additive on their graphs, Selected topics in functional equations (Graz, 1986), Ber. No. 292, Ber. Math.-Statist. Sekt. Forschungsgesellsch. Joanneum, Forschungszentrum Graz, Graz, 1988. MR 90j:39009

[118] W. JarczyK, On a problem of Z. Daróczy, Ann. Math. Sil. 5 (1991), 83-90. MR 93a:39015

[119] W. JARCZYK, A recurrent method of solving iterative functional equations, Prace Naukowe Uniwersytetu Ślạskiego w Katowicach 1206, Uniwersytet Ślạski, Katowice, 1991. MR 92k:39005

[120] W. JARCZYK, Globally attractive fixed points of continuous self-mappings of a real interval, European Conference on Iteration Theory (Batschuns, 1989), 157-170, World Sci. Publishing, River Edge, 1991. MR 93g:58070

[121] W. JARCZYK, On continuous solutions of the equation of invariant curves, Constantin Carathéodory: an international tribute, Vol. I, 527-542, World. Sci. Publishing, Teaneck, 1991. MR 92k:39012 
[122] W. JARCZYK, Convexity properties of nonnegative solutions of a convolution equation, Selected topics in functional equations and iteration theory (Graz, 1991), 71-92, Grazer Math. Ber. 316, Karl-Franzens-Univ. Graz, Graz, 1992. MR 94k:39046

[123] W. JARCZYK, On non-negative solutions of a convolution equation, Ann. Math. Sil. 8 (1994), 85-95. MR 96j:39031

[124] W. JARCZYK, On an equation of linear iteration in real and complex domains, Report of meeting. The Thirty-third International Symposium on Functional Equations (Caldes de Malavella, 1995), Aequationes Math. 51 (1996), 152. MR 98a:39023

[125] W. JarczyK, Problem 5.1, Report of meeting. The Thirty-third International Symposium on Functional Equations (Caldes de Malavella, 1995), Aequationes Math. 51 (1996), 163164.

[126] W. JARCZYK, On an equation of linear iteration, Aequationes Math. 51 (1996), 303-310. MR 98a:39023

[127] W. JARCZYK, Series of iterates summing up to the identity function, European Conference on Iteration Theory (Opava, 1994). Grazer Math. Ber. 334, 153-162, Karl-Franzens-Univ. Graz, Graz, 1997.

[128] W. JARCZYK, K. Łoskot AND M. C. Zdun, Commuting functions and simultaneous Abel equations, Ann. Polon. Math. 60 (1994), 119-135. MR 95k:39022

[129] W. JarczyK AND M. SABlik, Duplicating the cube and functional equations, Results Math. 26 (1994), 324-335. MR 96c:39016

[130] A. Joffe And F. Spitzer, On multitype branching processes with $\varrho \leq 1$, J. Math. Anal. Appl. 19 (1967), 409-430. MR 35\#3760

[131] A. S. Jones, On a conjecture of Mortell and Seymour, SIAM J. Appl. Math. 41 (1981), 107-111. MR 83j:39004

[132] P. Kahlig, A. Matkowska And J. Matkowski, On a class of a composite functional equations in a single variable, Aequationes Math. 52 (1996), 260-283. MR 97k:39017

[133] P. Kahlig And J. Smítal, On the solutions of a functional equation of Dhombres, Results Math. 27 (1995), 362-367. MR 96e:39010

[134] P. Kahlig And J. Smítal, On a parametric functional equation of Dhombres type, Aequationes Math. 56 (1998), 63-68. MR 99j:39019

[135] H.-H. KaIRIEs, Problem P225, Report of meeting. The Twenty-first International Symposium on Functional Equations (Konolfingen, 1983), Aequationes Math. 26 (1984), 271.

[136] H.-H. KaIRIES, Replicative Fourier series, Aequationes Math. 39 (1990), 281-282.

[137] H.-H. KAIRIEs, Replikativität und Funktionenräume, Preprint, Technische Universität Clausthal, Mathematik-Bericht 92/6.

[138] H.-H. KaIRIEs, Problem 27, Report of meeting. The Thirtieth International Symposium on Functional Equations (Oberwolfach, 1992), Aequationes Math. 46 (1993), 298.

[139] H.-H. KAIRIES, Die Gammafunktion als stetige Lösung eines Systems von Gauss-Funktionalgleichungen, Results Math. 26 (1994), 306-315. MR 95i:39013

[140] H.-H. KaIRIEs, $\mathrm{Zu}$ einem Characterisierungssatz von E. Artin, Abh. Math. Sem. Univ. Hamburg 65 (1995), 135-146. MR 97a:33002

[141] H.-H. KaIRIES, Problem 16, Report of meeting. The thirty-second International Symposium on Functional Equations (Gargnano, 1994), Aequationes Math. 49 (1995), 190.

[142] H.-H. KAIRIES, On the optimality of a characterization theorem for the gamma function using the multiplication formula, Aequationes Math. 51 (1996), 115-128. MR 97a:33003

[143] H.-H. KAIRIES, Functional equations for peculiar functions, Aequationes Math. 53 (1997), 207-241. MR 99d:39032

[144] H.-H. KAIRIES, Takagi's function and its functional equations, Rocznik Nauk.-Dydakt. Prace Mat. 15 (1998), 73-83.

[145] H.-H. KaIRIES, A remarkable system of eight functional equations, Aequationes Math. 58 (1999), 183-191.

[146] D. E. KNUTh, The art of computer programming, Vol. I: Fundamental algorithms. AddisonWesley Publishing Co., Reading, Mass.-London-Don Mills, 1969. MR 44\#3530

[147] P. Kostyrko, An abstract form of a conditional Cauchy's equation, Tatra Mt. Math. Publ. 2 (1993), 61-64. MR 94k:39058 
[148] M. Kuczma, Functional equations in a single variable, Monografie Mat. 46, Polish Scientific Publishers, Warsaw, 1968. MR 37\#4441

[149] M. Kuczma, On squares of differentiable functions, Ann. Polon. Math. 22 (1969), 229-237. MR 40\#7665

[150] M. KuCZMA, Almost convex functions, Colloq. Math. 21 (1970), 279-284. MR 41\#7043

[151] M. Kuczma, An introduction to the theory of functional equations and inequalities. Cauchy's equation and Jensen's inequality, Uniwersytet Śląski, Katowice; Polish Scientific Publishers, Warsaw, 1985. MR 86i:39008

[152] M. Kuczma, On a functional equation occuring in astrophysics, Math. Pannon. 3 (1992), 17-27. MR 94j:39011

[153] M. Kuczma, B. Choczewski And R. Ger, Iterative functional equations, Encyclopedia of Mathematics and Its Applications 32, Cambridge University Press, Cambridge, 1990. MR 92f:39002

[154] M. LaczKovich, Problem P211, Aequationes Math. 24 (1982), 291.

[155] M. LACZKOVICH, Nonnegative measurable solutions of difference equations, J. London Math. Soc. (2) 34 (1986), 139-147. MR 87m:39003

[156] M. LACZKOVICH, Operators commuting with translations, and systems of difference equations, Colloq. Math. 80 (1999), 1-22. MR 2000b:47082

[157] M. LaczKovich AND R. RedhefFer, Oscillating solutions of integral equations and linear recursions, Aequationes Math. 41 (1991), 13-32. MR 92e:45016

[158] M. H. LANTSMAN, Linear difference equations with coefficients of the power order of growth, Math. Nachr. 163 (1993), 305-322. MR 94k:39020

[159] Ka-Sing LaU ANd Jianrong Wang, Characterization of $L^{p}$-solutions for the two-scale dilation equations, SIAM J. Math. Anal. 26 (1995), 1018-1046. MR 96f:39004

[160] Ka-Sing LAU AND WeI-Bin Zeng, The convolution equation of Choquet and Deny on semigroups, Studia Math. 97 (1990), 115-135. MR 92b:43002

[161] D. Laugwitz and B. Rodewald, A simple characterization of the gamma function, Amer. Math. Monthly 94 (1987), 534-536. MR 89h:33003

[162] R. Leipnik And R. OberG, Subvex functions and Bohr's uniqueness theorem, Amer. Math. Monthly 74 (1967), 1093-1094. MR 37\#2922

[163] Z. LEŚNIAK, On homeomorphic and diffeomorphic solutions of the Abel equation on the plane, Ann. Polon. Math. 58 (1993), 7-18. MR 94f:39011

[164] Z. LEŚNIAK, On simultaneous Abel inequalities, Opuscula Math. 14 (1994), 107-115. MR 95j:39050

[165] Z. LEŚNIAK, On the system of the Abel equations on the plane, Ann. Math. Sil. 9 (1995), 105-122. MR 97b:39050

[166] Z. LEŚNIAK, Constructions of fractional iterates of Sperner homeomorphisms of the plane, European Conference on Iteration Theory (Batschuns, 1992), 182-192, World Sci. Publishing, River Edge, 1996. MR 98k:39010

[167] L. G. Lucht, Arithmetical aspects of certain functional equations, Acta Arith. 82 (1997), 257-277. MR 98m:11005

[168] L. G. Lucht, Arithmetical sequences and systems of functional equations, Aequationes Math. 53 (1997), 73-90. MR 98h:11007

[169] L. G. Lucht, Problem 12, Report of meeting. The Thirty-fifth International Symposium on Functional Equations (Graz-Mariatrost, 1997), Aequationes Math. 55 (1998), 301-302.

[170] St. Łojasiewicz, Solution générale de l'équation fonctionelle $f(f(. . f(x) .))=.g(x)$, Ann. Soc. Polon. Math. 24 (1951), 88-91. MR 14,285g

[171] M. MALEnicA, On the solutions of the functional equation $\varphi(x)+\varphi^{2}(x)=F(x)$, Mat. Vesnik 6 (19)(34) (1982), 301-305. MR 85g:39008

[172] M. MALENICA, Application of $\theta$-summability solving the functional equation $\varphi(x)+\varphi^{2}(x)$ $=F(x)$ [Serbo-Croatian], Akad. Nauka Umjet. Bosne Hercegov. Rad. Odjelj. Tehn. Nauka 10 (1986), 139-143. MR 87j:39016 
[173] J. Matkowski, On the functional equation $\varphi(x+\varphi(x))=\varphi(x)+\varphi(\varphi(x))$, Report of meeting. The Twenty-third International Symposium on Functional Equations (Gargnano, 1985), 27-28, University of Waterloo, Waterloo, 1985.

[174] J. MatKowski, Functions which are additive on their graphs and some generalizations, Rocznik Nauk.-Dydakt. Prace Mat. 13 (1993), 233-240. MR 96j:39022

[175] J. Matkowski, Remark 27, Report of meeting. The Thirty-first International Symposium on Functional Equations (Debrecen, 1993), Aequationes Math. 47 (1994), 319.

[176] J. MatKowski, On a system of simultaneous iterative functional equations, Ann. Math. Sil. 9 (1995), 123-135. MR 97a:39029

[177] J. Matkowski And M. SABlik, Some remarks on a problem of C. Alsina, Stochastica 10 (1986), 199-212. MR 89e:39011

[178] G. Mayor and J. Torrens, de Rham systems and the solution of a class of functional equations, Aequationes Math. 47 (1994), 43-49. MR 94k:39041

[179] P. J. MCCARTHY, Ultrafunctions, projective function geometry, and polynomial functional equations, Proc. London Math. Soc. (3) 53 (1986), 321-339. MR 88c:39009

[180] M. MERKLE, Conditions for convexity of a derivative and some applications to the gamma function, Aequationes Math. 55 (1998), 273-280. MR 99d:33002

[181] J. Milnor, On polylogarithms, Hurwith zeta functions, and the Kubert identities, Enseign. Math. (2) 29 (1983), 281-322. MR 86d:11007

[182] C. Mira ANd S. MüllenbaCh, Sur l'itération fractionnaire d'un endomorphisme quadratique, C. R. Acad. Sci. Paris Ser. I Math. 297 (1983), 369-372. MR 85e:58077

[183] D. S. MoAK, The q-gamma function for $q>1$, Aequationes Math. 20 (1980), 278-285. MR 81f:33002

[184] J. Morawiec, On bounded solutions of a problem of R. Schilling, Ann. Math. Sil. 8 (1994), 97-101. MR 96i:39025

[185] J. Morawiec, On a linear functional equation, Bull. Polish. Acad. Sci. Math. 43 (1995), 131-142. MR 98c:39009

[186] J. MoRAwIEC, On continuous solutions of a problem of R. Schilling, Results Math. 27 (1995), 381-386. MR 96i:39026

[187] J. MorawiEc, Bounded solutions of Schilling's problem, Math. Pannon. 7 (1996), 223-232. MR 97i:39009

[188] J. MoraWIEC, Some properties of probability distribution solutions of linear functional equations, Aequationes Math. 56 (1998), 81-90. MR 99m:39021

[189] J. MoRAWIEC, On locally bounded solutions of Schilling's problem, manuscript.

[190] J. MoraWIEc, On the existence of irregular solutions of the two-coefficient dilation equation, Aequationes Math. to appear.

[191] J. MoraWIEC AND J. WALORSKI, On the existence of smooth solutions of linear functional equations, Integral Equations Operator Theory.

[192] Z. Moszner, Problème P2, Aequationes Math. 1 (1968), 150.

[193] Z. Moszner, Remarque P283R1, Aequationes Math. 32 (1987), 146.

[194] Z. Moszner, Sur le prolongement covariant d'une équation linéaire par rapport au groupe d'itération, Sitzungsber. Abt. 2 (1998), 207; 173-182.

[195] A. MukherJea AND J. S. RATTI, On a functional equation involving iterates of a bijection on the unit interval, Nonlinear Anal. 7 (1983), 899-908; II. Nonlinear Anal. 31 (1998), 459-464. MR 84j:39007, 99d:39021

[196] F. Neuman, Global properties of linear ordinary differential equations, Mathematics and Its Applications (East European Series) 52, Kluwer Academic Publishers Group, Dordrecht, 1991. MR 94c:34010

[197] S. Ž. NIKČEVIĆ, Linear group functional equation and existence of the group inverse of the corresponding matrix, Facta Univ. Ser. Math. Inform. 5 (1990), 9-18. MR 92i:39011

[198] K. Nikodem, On E-invariant measures and a functional equation, Czechoslovak Math. J. 41(116) (1991), 565-569. MR 92m:39014

[199] S. Paganoni Marzegalli, One-parameter system of functional equations, Aequationes Math. 47 (1994), 50-59. MR 94k:39048 
[200] Yibiao Pan, On the existence of $L^{1}$ scaling functions, J. Math. Phys. Sci. 28 (1994), 55-74. MR 96b:39016

[201] Y. Peres AND B. Solomyak, Self-similar measures and intersections of Cantor sets, Trans. Amer. Math. Soc. 350 (1998), 4065-4087. MR 98m:26009

[202] A. O. Pittenger And J. V. RyfF, A probabilistic approach to scaling equations, Aequationes Math. 58 (1999), 242-259.

[203] T. PowierżA, Set-valued iterative square roots of bijections, Bull. Polish Acad. Sci. Math. 47 (1999), 377-383.

[204] T. PowIERŻA, On functions having weak iterative roots, manuscript.

[205] C. PraAgman, Fundamental solutions for meromorphic linear difference equations in the complex plane, and related problems, J. Reine Angew. Math. 369 (1986), 101-109. MR 88b:39004

[206] M. PyCIA, A convolution inequality, Aequationes Math. 57 (1999), 185-200. MR 2000h: 45004

[207] B. Ramachandran AND Ka-Sing LAU, Functional equations in probability theory, Probability and Mathematical Statistics, Academic Press, Inc., Boston, 1991. MR 93c:60016

[208] C. R. RaO And D. N. Shanbhag, An elementary proof for an extended version of the Choquet-Deny theorem, J. Multivariate Anal. 38 (1991), 141-148. MR 92j:60008

[209] C. R. RaO And D. N. Shanbhag, Some observations on the integrated Cauchy functional equation, Math. Nachr. 157 (1992), 185-195. MR 94k:62021

[210] C. R. RaO And D. N. Shanbhag, Choquet-Deny type functional equations with applications to stochastic models, John Wiley\&Sons, Chichester, 1994. MR 97d:60020

[211] TH. M. Rassias, Research problems on nonlinear equations, Nonlinear Analysis, 545-557, World Sci. Publishing, Singapore, 1987. MR 89c:00002

[212] J. S. Ratti And Y. F. Lin, A functional equation involving $f$ and $f^{-1}$, Colloq. Math. 60/61 (1990), 519-523. MR 92d:39016

[213] L. ReICH, Remark 24, Report of meeting. The Thirty-fifth International Symposium on Functional Equations (Graz-Mariatrost, 1997). Aequationes Math. 55 (1998), 311-312.

[214] Report of meeting. Second special session: Schilling's Problem, The Thirty-fifth International Symposium on Functional Equations (Graz-Mariatrost, 1997), Aequationes Math. 55 (1998), 314-315.

[215] G. DE RHAM, Sur quelques courbes definies par des equations fonctionnelles, Univ. e Politec. Torino. Rend. Sem. Mat. 16 (1956/57), 101-113. MR 20\#1733

[216] R. E. RICE, Iterative square roots of Čebyšev polynomials, Stochastica 3 (1979), 1-14. MR $80 \mathrm{~m}: 39005$

[217] R. E. Rice, B. Schweizer And A. Sklar, When is $f(f(z))=a z^{2}+b z+c$ ?, Amer. Math. Monthly 87 (1980), 252-263. MR 81e:39005

[218] H. Rüssmann, Über die Iteration analytischer Funktionen, J. Math. Mech. 17 (1967), 523532. MR 36\#1621

[219] M. Sablik, Problem 221, Aequationes Math. 26 (1983), 266.

[220] M. SABlik, Note on a Cauchy conditional equation, Rad. Mat. 1 (1985), 241-245. MR 87e:39015

[221] M. SABLIK, Some remarks on Cauchy equation on a curve, Demonstratio Math. 23 (1990), 477-490. MR 92d:39021

[222] M. Sablik, Differentiable solutions of functional equations in Banach spaces, Ann. Math. Sil. 7 (1993), 17-55. MR 95e:39006

[223] M. SABliK, A note on simultaneous Abel equations, In: W. Förg-Rob et al. (Eds.): Iteration Theory (Proceedings of the European Conference, Batschuns, 1992), 245-250, World Sci. Publishing, River Edge, 1996. MR 98m:39081

[224] J. Saint-RAYmond, Sur l'équation fonctionnelle $f(x)=f\left(\frac{1}{2}(x+1)\right)+f\left(\frac{1}{2}(x-1)\right)$, Initiation Seminar on Analysis: G. Choquet - M. Rogalski - J. Saint-Raymond, 19th Year: 1979/1980, Exp. No. 10, Publ. Math. Univ. Pierre et Marie Curie, 41, Univ. Paris VI, Paris, 1980. MR $84 \mathrm{j}: 39006$

[225] R. Schilling, Spatially chaotic structures, Nonlinear Dynamics in Solids, 213-241, SpringerVerlag, Berlin, 1992. 
[226] P. Schroth, On $\left(1, b_{p}\right)$-replicative functions with isolated discontinuities, Aequationes Math. 20 (1980), 73-79. MR 81k:39008

[227] B. SCHWEIZER AND A. SKLAR, Invariants and equivalence classes of polynomials under linear conjugacy, Contributions to general algebra 6, 253-257, Holder-Pichler-Tempsky, Vienna, 1988. MR 92d:12003

[228] S. L. Segal, On Nathanson's functional equation, Aequationes Math. 28 (1985), 114-123. MR 86d:39010

[229] S. L. SEGAL, Iterative characterizations of powers and exponentials, Aequationes Math. 37 (1989), 201-218. MR 90j:30042

[230] C. Sempi, Problem 28, Report of meeting. The Thirty-second International Symposium on Functional Equations (Gargnano, 1994), Aequationes Math. 49 (1995), 195.

[231] B. R. Seymour And M. P. Mortell, Discontinuous solutions of a measure-preserving mapping, SIAM J. Appl. Math. 41 (1981), 94-106. MR 83j:58100

[232] D. N. Shanbhag, Extended versions of Deny's theorem via de Finetti's theorem, Comput. Statist. Data Anal. 12 (1991), 115-126. MR 92m:60015

[233] J. H. ShaPIRo, Composition operators and Schröder's functional equation, Studies on composition operators (Laramie, WY, 1996), 213-228, Contemp. Math. 213, Amer. Math. Soc., Providence, 1998. MR 98m:47048

[234] Jian Guo SI, The $C^{2}$-solutions to the iterated equation $\sum_{i=1}^{n} \lambda_{i} f^{i}(x)=F(x)$ [Chinese], Acta Math. Sinica 36 (1993), 348-357. MR 94k:39042

[235] Jian Guo Si, On analytic solutions of the equation of invariant curves, C.R. Math. Rep. Acad. Sci. Canada 17 (1995), 49-52. MR 96e:39016

[236] A. Simon And P. Volkmann, Croissance d'une suite définie par certaine solution distribution d'une équation fonctionnelle, http://www.mathematik.uni-karlsruhe.de/ ${ }^{\sim}$ semlv, Sem. LV 2 (1998).

[237] K. Simon, Some dual statements concerning Wiener measure and Baire category, Proc. Amer. Math. Soc. 106 (1989), 455-463. MR 89k:26003

[238] K. Simon, Typical continuous functions are not iterates, Acta Math. Hungar. 55 (1990), 133-134. MR 91m:26005

[239] A. SMAJdor AND W. SMAJdor, Multivalued solutions of a functional equation, Ann. Polon. Math. 41 (1983), 89-97. MR 85d:39009

[240] W. Smajdor, Analytic solutions of Schröder equation, Publ. Math. Debrecen 26 (1979), 149-153. MR 81f:39012

[241] W. SMAJdor, Multivalued solutions of a linear functional equation, Ann. Polon. Math. 45 (1985), 253-259. MR 87i:39020

[242] N. Steinmetz, Rational iteration. Complex analytic dynamical systems, de Gruyter Studies in Mathematics 16, Walter de Gruyter \& Co., Berlin, 1993. MR 94h:30035

[243] G. Strang, Wavelet transforms versus Fourier transforms, Bull. Amer. Math. Soc. (N.S.) 28 (1993), 288-305. MR 94b:42017

[244] JACEK TABOR, Remark 6, Report of meeting. The Thirty-third International Symposium on Functional Equations (Caldes de Malavella, 1995), Aequationes Math. 51 (1996), 164.

[245] JACEK TABOR, Problem 14, Report of meeting. The Thirty-third International Symposium on Functional Equations (Caldes de Malavella, 1995), Aequationes Math. 51 (1996), 170.

[246] JACEK TABOR, Linear difference equations and invariant subsets of linear mappings, Aequationes Math. 57 (1999), 153-184. MR 2000g:39008

[247] JACEK TABOR AND Józef TABOR, On a linear iterative equation, Results Math. 27 (1995), 412-421. MR 96k:39030

[248] Gy. Targonski, Problem 14.1, Report of meeting. Twenty-eight International Symposium on Functional Equations (Graz-Mariatrost, 1990), Aequationes Math. 41 (1991), 289.

[249] Gy. Targonski, Progress of iteration theory since 1981, Aequationes Math. 50 (1995), 50-72. MR 96i:39028 
[250] M. S. Tomás, On some iteration problems concerning sections of t-norms, European Conference on Iteration Theory (Caldes de Malavella, 1987), 453-456, World Sci. Publishing, Teaneck, 1989. MR 92c:39014

[251] P. Volkmann, Sur un système d'inéquations fonctionnelles, C. R. Math. Rep. Acad. Sci. Canada 4 (1982), 155-158. MR 83k:39010

[252] P. Volkmann And H. Weigel, Sur la solution de certaines équations fonctionnelles, Uniw. Ślasski w Katowicach Prace Nauk.-Prace Mat. 12 (1982), 22-29. MR 83g:39005

[253] P. L. WALker, A class of functional equations which have entire solutions, Bull. Austral. Math. Soc. 38 (1988), 351-356. MR 89j:39009

[254] P. L. Walker, The exponential of iteration of $e^{x}-1$, Proc. Amer. Math. Soc. 110 (1990), 611-620. MR 91b:58211

[255] P. L. Walker, On the solutions of an abelian functional equation, J. Math. Anal. Appl. 155 (1991), 93-110. MR 92e:39026

[256] J. WALORSKI, On some solutions of the Schröder equation in Banach spaces, Ann. Math. Sil. 8 (1994), 103-110. MR 96i:39036

[257] J. WALORSKI, An application of the Krein-Rutman theorem to the Abel equation, Bull. Polish. Acad. Sci. Math. 42 (1994), 217-228.

[258] J. WALORSKI, Convex solutions of the Schröder equation in Banach spaces, Proc. Amer. Math. Soc. 125 (1997), 153-158. MR 97c:39020

[259] B. WARE, The Siegel centerstable theorem in Hilbert space, Berichte der Mathemat.-Statist. Sekt. Forschungszentrum Graz 37 (1975), Forschungszentrum Graz, Graz, 1975. MR 58 \#13179

[260] R. Webster, Convexity, Oxford Science Publications. The Clarendon Press, Oxford University Press, New York, 1994. MR 98h:52001

[261] R. WebsteR, Log-convex solutions to the functional equation $f(x+1)=g(x) f(x)$ : $\Gamma$-type functions, J. Math. Anal. Appl. 209 (1997), 605-623. MR 98m:39072

[262] B. Weiss, Measurable dynamics, Conference in modern analysis and probability (New Haven, Conn., 1982), 395-421, Contemp. Math. 26, Amer. Math. Soc., Providence, 1984. MR 85j:28027

[263] G. J. Wirsching, The dynamical system generated by the $3 n+1$ function, Lecture Notes in Math. 1681, Springer-Verlag, Berlin, 1998. MR 99g:11027

[264] J.-C. YocCOZ, Linéarisation des germes de difféomorphismes holomorphes de ( $\mathbb{C}, 0)$, C.R. Acad. Sci. Paris Ser. I Math. 306 (1988), 55-58. MR 89i:58123

[265] J.-C. Yoccoz, Théorème de Siegel, nombres de Bruno et polynômes quadratiques, Astérisque 231 (1995), 3-88. MR 96m:58214

[266] M. F. Yoder, Continuous replicative functions, Aequationes Math. 13 (1975), 251-261. MR 53\#8706

[267] M. ZDUN, On the uniqueness of solutions of the functional equation $\varphi(x+f(x))=\varphi(x)+$ $\varphi(f(x))$, Aequationes Math. 8 (1972), 229-232. MR 47\#3869

[268] M. C. Zdun, Note on commutable functions, Aequationes Math. 36 (1988), 153-164. MR $89 \mathrm{~m}: 39012$

[269] M. C. Zdun, On simultaneous Abel equations, Aequationes Math. 38 (1989), 163-177. MR 90m:39019

[270] M. C. ZDUn, On diffeomorphic solutions of simultaneous Abel's equations, Arch. Math. (Brno) 27A (1991), 123-131. MR 94a:39014

[271] M. C. Zdun, On iterative roots of homeomorphisms of the circle, Bull. Polish Acad. Sci. Math. 48 (2000), 203-213.

[272] E. Zehnder, A simple proof of a generalization of a Theorem by C. L. Siegel, Geometry and topology (Proc. III Latin Amer. School of Math., Inst. Mat. Pura Aplicada CNPq, Rio de Janeiro, 1976), 855-866, Lecture Notes in Math. 597, Springer, Berlin, 1977. MR $57 \# 1560$

[273] WeI-Bin Zeng, Two problems related to the integrated Cauchy functional equation, Report of meeting. The Twenty-nineth International Symposium on Functional Equations (Wolfville, 1991), Aequationes Math. 43 (1992), 299-300. 
[274] WeI-Bin Zeng, Problem 27, Report of meeting. The Twenty-ninth International Symposium on Functional Equations (Wolfville, 1991), Aequationes Math. 43 (1992), 306.

[275] Jingzhong Zhang, Lu Yang And Weinian Zhang, Some advances on functional equations, Adv. in Math. (China) 24 (1995), 385-405. MR 97d:39009

[276] Weinian Zhang, Stability of the solution of the iterated equation $\sum_{i=1}^{n} \lambda_{i} f^{i}(x)=F(x)$, Acta Math. Sci. (English Ed.) 8 (1988), 421-424. MR 90e:39010

[277] WeInIAN ZHANG, On the differentiable solutions of the iterated equation $\sum_{i=1}^{n} \lambda_{i} f^{i}(x)=F(x)$ [Chinese], Acta Math. Sinica 32 (1989), 98-109. MR 90i:39009

[278] Weinian Zhang, Discussion on the differentiable solutions of the iterated equation $\sum_{i=1}^{n} \lambda_{i} f^{i}(x)=F(x)$, Nonlinear Anal. 15 (1990), 387-398. MR 92a:39010

[279] LI Ren ZhaO, Existence and uniqueness theorem for the solution of the functional equation $\lambda_{1} f(x)+\lambda_{2} f^{2}(x)=F(x)$ [Chinese], J. China Univ. Sci. Tech. 13 (1983), Math. Issue, 21-27. MR 86b:39006

[280] JiAn HuA ZHENG, Nonexistence of admissible solution of the function equation $\sum_{k=0}^{p} a_{k} f^{n_{k}}=$ 1, Dongbei Shuxue 6 (1990), 60-64. MR 92c:39021

[281] Wang Jian Zhong, On solutions of two-scale difference equations, Chinese Ann. Math. 15 (1994), 23-34. MR 95b:39005

K. Baron

Instytut Matematyki

Uniwersytet Śląski

ul. Bankowa 14

PL-40-007 Katowice

Poland

\author{
W. Jarczyk \\ Instytut Matematyki \\ Uniwersytet Śląski \\ ul. Bankowa 14 \\ PL-40-007 Katowice \\ Poland
}

and

Instytut Matematyki

Politechnika Zielonagórska

ul. Podgórna 50

PL-65-246 Zielona Góra

Poland

Manuscript received: October 11, 1999 and, in final form, May 15, 2000. 